\title{
Observer Design for Delayed Markovian Jump Systems with Output State Saturation
}

\author{
Guoliang Wang (iD) and Bo Feng \\ School of Information and Control Engineering, Liaoning Shihua University, Fushun, Liaoning 113001, China \\ Correspondence should be addressed to Guoliang Wang; gliangwang@aliyun.com
}

Received 16 June 2017; Accepted 17 December 2017; Published 22 January 2018

Academic Editor: Renming Yang

Copyright (C) 2018 Guoliang Wang and Bo Feng. This is an open access article distributed under the Creative Commons Attribution License, which permits unrestricted use, distribution, and reproduction in any medium, provided the original work is properly cited.

This paper considers the observer design problem of continuous-time delayed Markovian jump systems with output state saturation. Different from the traditionally observer-based saturation control methods, a kind of system output state saturation with a partially delay-dependent property is proposed, where both nondelay and delay states exist at the same time but happen asynchronously. By exploiting the Bernoulli variable, the probability distributions of such two states are described and considered in the observer design. Based on an improved equality applied to deal with saturation terms, sufficient conditions for the designed observer with three kinds of output saturations are all provided with LMI forms. Finally, a numerical example is given to indicate the effectiveness of the obtained results.

\section{Introduction}

As we know, Markovian jump system (MJS) is a special kind of stochastic hybrid dynamical systems. Because of two kinds of mechanisms contained, it is very suitable to model such actual systems whose structures or parameters change $[1,2]$. Over the past years, many research topics on MJSs have been extensively studied, like stability analysis [3-6], stabilization [7-11], robust control [12-15], adaptive control [16-19], $H_{\infty}$ filtering and control $[20,21]$, state estimation [22-25], synchronization [26-30], and so on.

On the other hand, saturation problem is commonly encountered in many physical systems. It is mainly because the transmitted signal is usually affected by environmental factors, physical factors, and even technical factors. When signal occurs with saturation, the system performance will be reduced. Therefore, the effect of saturation is necessarily studied, as in [31-35]. By investigating the above results, it is found that they mainly focused on the estimate of domain of attraction, in which an equivalent description of saturation actuator is necessary. However, the introduction of this transformation will make the computation complexity very large, especially for an MJS with $N$ operation modes. Recently, one inequality was introduced in [36] and applied to deal with saturation. In the modern control theory, it is obvious that the state feedback control has advantages in solving the problems of system stability, pole placement, stabilization, and optimal control. However, system state usually cannot be measured directly, or the measurement is limited by objective conditions. In this case, it is not easy to achieve the object by state feedback control. The better way is to make full use of system output. When output is saturated, some results were presented in $[37,38]$. By investigating these references, it is found that there are still many problems to be considered. For example, there is no time delay in the saturated output, while time delay is usually associated with most system states and could lead to unstable system. Thus, it is necessary to consider them together. To our best knowledge, very few results are available to study the above problems. All observations motivate current research.

In this paper, the observer design problem of continuoustime delayed Markovian jump systems with output state saturation is studied, where the output state saturation is partially delay-dependent. The main contributions of this paper are generalized as follows: (1) A kind of observer based on partially delay-dependent output is proposed. Here, both nondelay and delay states are contained in output saturation simultaneously, but their occurrences are asynchronous. (2) 
The probability distributions of such two states are embodied by the Bernoulli variable and fully considered in the observe design. (3) Sufficient conditions of the designed observer are obtained by applying an improved inequality to deal with the saturation terms. In order to make the computation solved easily and directly, the existence conditions are presented within LMI framework by introducing some additional variables and inequalities. (4) Based on the proposed methods and techniques, another two kinds of observers based on different output saturations are proposed, whose conditions are all LMIs.

Notation $1 . \mathbb{R}^{n}$ denotes the $n$-dimensional Euclidean space; $\mathbb{R}^{q \times n}$ is the set of all $q \times n$ real matrices. $\|\cdot\|$ refers to the Euclidean vector norm or spectral matrix norm. $\Omega$ is the sample space, $\mathscr{F}$ is the $\sigma$-algebra of subsets of the sample space, and $\mathbb{P}$ is the probability measure on $\mathscr{F}$. In symmetric block matrices, we use “*” as an ellipsis for the terms induced by symmetry, $\operatorname{diag}\{\cdots\}$ for a block-diagonal matrix, and $(M)^{\star} \triangleq M+M^{T}$.

\section{Problem Formulation}

Consider the following continuous-time delayed Markovian jump systems with output state saturation:

$$
\begin{aligned}
& \dot{x}(t)=A\left(r_{t}\right) x(t), \\
& y(t)=C\left(r_{t}\right) \text { sat }[\alpha(t) x(t)+(1-\alpha(t)) x(t-d)], \\
& x(t)=\phi(t), \quad-d \leq t \leq 0,
\end{aligned}
$$

where $x(t) \in \mathbb{R}^{n}$ is the state vector, $y(t) \in \mathbb{R}^{q}$ is the output, and $\phi(t) \in \mathbb{R}^{n}$ is the initial condition. Matrices $A\left(r_{t}\right)$ and $C\left(r_{t}\right)$ are known matrices of compatible dimensions. $\left\{r_{t}, t \geq\right.$ $0\}$ is a continuous-time Markov process taking values in a finite set $\mathbb{S}=\{1,2, \ldots, N\}$ with transition rate matrix (TRM) $\Pi \triangleq\left(\pi_{i j}\right) \in \mathbb{R}^{N \times N}$ given by

$$
\operatorname{Pr}\left\{r_{t+\Delta t}=j \mid r_{t}=i\right\}= \begin{cases}\pi_{i j} \Delta t+o(\Delta t), & i \neq j \\ 1+\pi_{i i} \Delta t+o(\Delta t), & i=j,\end{cases}
$$

where $\Delta t>0, \pi_{i j} \geq 0$, if $i \neq j$, and $\pi_{i i}=-\sum_{j \neq i} \pi_{i j}$ for all $i, j \in \mathbb{S}$. Time delay $d$ satisfies $d>0$, and $\alpha(t)$ is the Bernoulli stochastic variable and defined as

$$
\alpha(t)= \begin{cases}1, & \text { if } x(t) \text { is available } \\ 0, & \text { if } x(t-d) \text { is available. }\end{cases}
$$

It is satisfied as

$$
\begin{aligned}
& \operatorname{Pr}\{\alpha(t)=1\}=\mathscr{E}\{\alpha(t)\}=\alpha, \\
& \operatorname{Pr}\{\alpha(t)=0\}=1-\alpha .
\end{aligned}
$$

Function sat( $\cdot): \mathbb{R}^{n} \rightarrow \mathbb{R}^{n}$ is the standard saturation function and defined as

$$
\operatorname{sat}\left(u_{i}\right)= \begin{cases}\mu_{i}, & u_{i}>\mu_{i} \\ u_{i}, & -\mu_{i} \leq u_{i} \leq \mu_{i} \quad i \in 1,2, \ldots, n, \\ -\mu_{i}, & u_{i}<-\mu_{i},\end{cases}
$$

where

$$
\begin{aligned}
\operatorname{sat}(u) & =\left[\begin{array}{lll}
\operatorname{sat}\left(u_{1}\right) & \cdots & \operatorname{sat}\left(u_{n}\right)
\end{array}\right]^{T}, \\
\mu & =\left[\begin{array}{lll}
\mu_{1} & \cdots & \mu_{n}
\end{array}\right]^{T} .
\end{aligned}
$$

Remark 1. It is worth mentioning that saturation in output is more general and has some advantages. Firstly, compared with saturations described in [36, 39-42] where nondelay state should be available online, system state $x(t)$ in output is not necessary and could be replaced by delay state $x(t-d)$. Secondly, compared with the saturated controllers designed for delayed systems without delay state $[43,44]$, both nondelay and delay states are included in output, whose distribution probabilities are also considered. Because all the delay terms could affect delayed systems, the obtained results are more conservative without considering them. In this sense, it is said that our formulation about output saturation has a larger application scope and less conservatism.

By letting $\eta=x-\operatorname{sat}(x)$ and $\eta_{d}=x_{d}-\operatorname{sat}\left(x_{d}\right)$ and considering the definition of $\alpha(t)$, it is known that

$$
\begin{aligned}
\text { sat }[\alpha(t) x(t)+(1-\alpha(t)) x(t-d)] \\
=\alpha(t) x(t)+(1-\alpha(t)) x(t-d) \\
\quad-\left[\alpha(t) \eta+(1-\alpha(t)) \eta_{d}\right] \\
=\alpha x(t)+(1-\alpha) x(t-d) \\
\quad+(\alpha(t)-\alpha)(x(t)-x(t-d)) \\
\quad-\left[\alpha \eta+(1-\alpha) \eta_{d}+(\alpha(t)-\alpha)\left(\eta-\eta_{d}\right)\right] .
\end{aligned}
$$

Then, system (1) is rewritten to be

$$
\begin{aligned}
\dot{x}(t) & =A\left(r_{t}\right) x(t), \\
y(t) & =C\left(r_{t}\right)\{\alpha x(t)+(1-\alpha) x(t-d) \\
+ & (\alpha(t)-\alpha)(x(t)-x(t-d)) \\
- & {\left.\left[\alpha \eta+(1-\alpha) \eta_{d}+(\alpha(t)-\alpha)\left(\eta-\eta_{d}\right)\right]\right\}, } \\
x(t) & =\phi(t), \quad-d \leq t \leq 0 .
\end{aligned}
$$

In this paper, the designed state observer system is described by

$$
\begin{aligned}
& \dot{\hat{x}}(t)=A\left(r_{t}\right) \hat{x}(t)+G\left(r_{t}\right)(y(t)-\widehat{y}(t)), \\
& \widehat{y}(t)=C\left(r_{t}\right) \hat{x}(t),
\end{aligned}
$$

where $\widehat{x}(t) \in \mathbb{R}^{n}$ is the estimation of $x(t)$, and $\widehat{y}(t) \in \mathbb{R}^{q}$ is the corresponding output. Define $e(t)=x(t)-\widehat{x}(t)$; the resulting error system is rewritten to be

$$
\begin{aligned}
\dot{e}(t)= & \left(A\left(r_{t}\right)-G\left(r_{t}\right) C\left(r_{t}\right)\right) e(t) \\
& +(1-\alpha) G\left(r_{t}\right) C\left(r_{t}\right) x(t)
\end{aligned}
$$




$$
\begin{aligned}
& -(1-\alpha) G\left(r_{t}\right) C\left(r_{t}\right) x(t-d) \\
& +\alpha G\left(r_{t}\right) C\left(r_{t}\right) \eta+(1-\alpha) G\left(r_{t}\right) C\left(r_{t}\right) \eta_{d} \\
& +(\alpha(t)-\alpha) G\left(r_{t}\right) C\left(r_{t}\right) \\
& \times\left(x(t-d)-x(t)+\eta-\eta_{d}\right) .
\end{aligned}
$$

Here, it is assumed that stochastic variables $\alpha(t)$ and $r_{t}$ are independent.

Proposition 2. Let $u=\alpha(t) K x(t)+(1-\alpha(t)) K_{d} x(t-d)$ and $\eta=u-\operatorname{sat}(u)$. Then, there exist real numbers $\varepsilon \in(0,1)$ and $\varepsilon_{d} \in(0,1)$ such that

$$
\begin{aligned}
\eta^{T} \eta \leq & \alpha(t) \varepsilon x^{T}(t) K^{T} K x(t) \\
& +(1-\alpha(t)) \varepsilon_{d} x^{T}(t-d) K_{d}^{T} K_{d} x(t-d),
\end{aligned}
$$

where $\eta=\left[\begin{array}{lll}\eta_{1} & \cdots & \eta_{n}\end{array}\right]^{T}$, and $\eta_{i}$ is the dead-zone nonlinearity function, $i=\{1,2, \ldots, n\}$.

Proof. Based on the formulation of $u(t)$, it is obtained that

$$
\begin{aligned}
\eta^{T} \eta & =\left\{\alpha(t) K x(t)+(1-\alpha(t)) K_{d} x(t-d)\right. \\
& \left.-\operatorname{sat}\left[\alpha(t) K x(t)+(1-\alpha(t)) K_{d} x(t-d)\right]\right\}^{T} \\
& \cdot\left\{\alpha(t) K x(t)+(1-\alpha(t)) \times K_{d} x(t-d)\right. \\
& \left.-\operatorname{sat}\left[\alpha(t) K x(t)+(1-\alpha(t)) K_{d} x(t-d)\right]\right\} .
\end{aligned}
$$

On the one hand, if $\alpha(t)=1$, one has $u=K x(t)$. Similar to [36], we have the following.

(i) If $u_{i}>\mu_{i}$, it is obtained that

$$
\begin{aligned}
\eta^{T} \eta= & (K x(t)-\operatorname{sat}(K x(t)))^{T}(K x(t)-\operatorname{sat}(K x(t))) \\
= & x^{T}(t) K^{T} K x(t)-2 x^{T}(t) K^{T} \text { sat }(K x(t)) \\
& +(\operatorname{sat}(K x(t)))^{T}(\operatorname{sat}(K x(t))) \\
= & x^{T}(t) K^{T} K x(t)-2 x^{T}(t) K^{T} \mu+\mu^{T} \mu \\
< & x^{T}(t) K^{T} K x(t)-\mu^{T} \mu \leq \varepsilon x^{T}(t) K^{T} K x(t)
\end{aligned}
$$

where $\varepsilon \in(0,1)$ could be selected.

(ii) If $u_{i}<-\mu_{i}$, it is found that

$$
\begin{aligned}
\eta^{T} \eta= & (K x(t)-\operatorname{sat}(K x(t)))^{T}(K x(t)-\operatorname{sat}(K x(t))) \\
= & x^{T}(t) K^{T} K x(t)-2 x^{T}(t) K^{T} \text { sat }(K x(t)) \\
& +(\operatorname{sat}(K x(t)))^{T}(\operatorname{sat}(K x(t))) \\
= & x^{T}(t) K^{T} K x(t)+2 x^{T}(t) K^{T} \mu+\mu^{T} \mu \\
< & x^{T}(t) K^{T} K x(t)-\mu^{T} \mu \leq \varepsilon x^{T}(t) K^{T} K x(t)
\end{aligned}
$$

where $\varepsilon \in(0,1)$ could be obtained too. (iii) If $-\mu_{i} \leq u_{i} \leq \mu_{i}$, we have $\eta^{T} \eta=0$. Then, there is always a scalar $\varepsilon \in(0,1)$ such that

$$
\eta^{T} \eta \leq \varepsilon x^{T}(t) K^{T} K x(t)
$$

On the other hand, when $\alpha(t)=0$, it is obtained that $u=$ $K_{d} x(t-d)$. Similarly, we have the following cases.

(i) If $u_{i}>\mu_{i}$, it is obtained that

$$
\begin{aligned}
\eta^{T} \eta & =\left\{K_{d} x(t-d)-\text { sat }\left[K_{d} x(t-d)\right]\right\}^{T} \\
\cdot & \left\{K_{d} x(t-d)-\operatorname{sat}\left[K_{d} x(t-d)\right]\right\}=x^{T}(t-d) \\
\cdot & K_{d}^{T} K_{d} x(t-d)-2 x^{T}(t) K_{d}^{T} \operatorname{sat}\left[K_{d} x(t-d)\right] \\
& +\left(\operatorname{sat}\left[K_{d} x(t-d)\right]\right)^{T} \operatorname{sat}\left[K_{d} x(t-d)\right] \\
& =x^{T}(t-d) K_{d}^{T} K_{d} x(t-d)-2 x^{T}(t-d) K_{d}^{T} \mu \\
& +\mu^{T} \mu<x^{T}(t-d) K_{d}^{T} K_{d} x(t-d)-\mu^{T} \mu \\
& \leq \varepsilon_{d} x^{T}(t-d) K_{d}^{T} K x(t-d),
\end{aligned}
$$

where $\varepsilon_{d} \in(0,1)$.

(ii) If $u_{i}<-\mu_{i}$, it is found that

$$
\begin{aligned}
\eta^{T} \eta & =\left\{K_{d} x(t-d)-\text { sat }\left[K_{d} x(t-d)\right]\right\}^{T} \\
\cdot & \left\{K_{d} x(t-d)-\operatorname{sat}\left[K_{d} x(t-d)\right]\right\}=x^{T}(t-d) \\
\cdot & K_{d}^{T} K_{d} x(t-d)-2 x^{T}(t) K_{d}^{T} \text { sat }\left[K_{d} x(t-d)\right] \\
& +\left(\operatorname{sat}\left[K_{d} x(t-d)\right]\right)^{T} \operatorname{sat}\left[K_{d} x(t-d)\right] \\
& =x^{T}(t-d) K_{d}^{T} K_{d} x(t-d)+2 x^{T}(t-d) K_{d}^{T} \mu \\
& +\mu^{T} \mu<x^{T}(t-d) K_{d}^{T} K_{d} x(t-d)-\mu^{T} \mu \\
& \leq \varepsilon_{d} x^{T}(t-d) K_{d}^{T} K x(t-d),
\end{aligned}
$$

where $\varepsilon_{d} \in(0,1)$.

(iii) If $-\mu_{i} \leq u_{i} \leq \mu_{i}$, we have $\eta^{T} \eta=0$. Then, there is always a scalar $\varepsilon_{d} \in(0,1)$ such that

$$
\eta^{T} \eta \leq \varepsilon_{d} x^{T}(t-d) K_{d}^{T} K_{d} x(t-d) .
$$

This completes the proof.

\section{Main Results}

Theorem 3. Given system (1) with $d>0$, there exists an observer (9) such that system (1) and error system (12) are asymptotically stable, if for given positive scalars $\alpha \in[0,1]$, $\varepsilon_{i} \in(0,1)$, and $\varepsilon_{d i} \in(0,1)$, there exist $P_{i}>0, Q>0, Z>$ $0, \gamma_{i}>0, \lambda_{i}>0$, and $Y_{i}$ such that 


$$
\begin{gathered}
{\left[\begin{array}{cccccc}
\Theta_{i 1} & \Theta_{i 2} & Z & 0 & \Theta_{i 3} & \Theta_{i 4} \\
* & \Theta_{i 5} & \Theta_{i 6} & Z & 0 & \Theta_{i 7} \\
* & * & \Theta_{i 8} & 0 & \Theta_{i 9} & \Theta_{i 10} \\
* & * & * & \Theta_{11} & 0 & 0 \\
* & * & * & * & \Theta_{i 12} & 0 \\
* & * & * & * & * & \Theta_{i 12}
\end{array}\right]<0,} \\
\\
\end{gathered}
$$

where

$$
\begin{aligned}
\Theta_{i 1}= & \left(P_{i} A_{i}\right)^{\star}+\sum_{j=1}^{N} \pi_{i j} P_{j}+\alpha \varepsilon_{i} \gamma_{i} I+Q+d^{2} A_{i}^{T} Z A_{i} \\
& -Z+2 \alpha d^{2} \varepsilon_{i} \lambda_{i} I+4 \alpha \bar{\alpha} d^{2} \varepsilon_{i} \lambda_{i} I, \\
\Theta_{i 2}= & \bar{\alpha} C_{i}^{T} Y_{i}^{T}, \\
\Theta_{i 3}= & -\sqrt{2 \alpha \bar{\alpha}} d C_{i}^{T} Y_{i}^{T}, \\
\Theta_{i 4}= & \sqrt{2} \bar{\alpha} d C_{i}^{T} Y_{i}^{T}, \\
\Theta_{i 5}= & \left(P_{i} A_{i}-Y_{i} C_{i}\right)^{\star}+P_{i}+\sum_{j=1}^{N} \pi_{i j} P_{j}+Q-Z, \\
\Theta_{i 6}= & -\bar{\alpha} Y_{i} C_{i}, \\
\Theta_{i 7}= & \sqrt{2} d\left(A_{i}^{T} P_{i}-C_{i}^{T} Y_{i}^{T}\right), \\
\Theta_{i 8}= & \bar{\alpha} \varepsilon_{d i} \gamma_{i} I+2 \bar{\alpha} d^{2} \varepsilon_{d i} \lambda_{i} I+4 \alpha \bar{\alpha} d^{2} \varepsilon_{d i} \lambda_{i} I-Q-Z, \\
\Theta_{i 9}= & \sqrt{2 \alpha \bar{\alpha}} d C_{i}^{T} Y_{i}^{T}, \\
\Theta_{i 10}= & -\sqrt{2} \bar{\alpha} d C_{i}^{T} Y_{i}^{T}, \\
\Theta_{11}= & -Q-Z, \\
\Theta_{i 12}= & -2 P_{i}+Z, \\
\bar{\alpha}= & 1-\alpha .
\end{aligned}
$$

Then, the gain of observer (9) is computed by

$$
G_{i}=Y_{i} P_{i}^{-1}
$$

Proof. For systems (1) and (12), choose the following Lyapunov function:

$$
V\left(x_{t}\right)=V_{1}\left(x_{t}\right)+V_{2}\left(x_{t}\right) \text {, }
$$

where

$$
\begin{aligned}
V_{1}\left(x_{t}, r_{t}\right)= & x^{T}(t) P\left(r_{t}\right) x(t)+\int_{t-d}^{t} x^{T}(s) Q x(s) d s \\
& +d \int_{-d}^{0} \int_{t+\theta}^{t} \dot{x}^{T}(s) Z \dot{x}(s) d s d \theta \\
V_{2}\left(x_{t}, r_{t}\right)= & e^{T}(t) P\left(r_{t}\right) e(t)+\int_{t-d}^{t} e^{T}(s) Q e(s) d s \\
& +d \int_{-d}^{0} \int_{t+\theta}^{t} \dot{e}^{T}(s) Z \dot{e}(s) d s d \theta .
\end{aligned}
$$

Let $\mathscr{L}$ be the weak infinitesimal generator of random process $\left\{x_{t}, r_{t}\right\}$, for each $r_{t}=i \in \mathbb{S}$; it is defined as

$$
\begin{aligned}
& \mathscr{L} V\left(x_{t}, t, i\right)=\lim _{\Delta t \rightarrow 0^{+}} \\
& \quad \cdot \frac{1}{\Delta t}\left\{\mathscr{E}\left[V\left(x_{t+\Delta t}, r_{t+\Delta t}, t+\Delta t\right) \mid x_{t}, r_{t}=i\right]\right. \\
& \left.\quad-V\left(x_{t}, i, t\right)\right\} .
\end{aligned}
$$

Then, it is further obtained that

$$
\begin{aligned}
& \mathscr{L} V_{1}\left(x_{t}\right) \\
&=x^{T}(t)\left(P_{i} A_{i}\right)^{\star} x(t)+x^{T}(t) \sum_{j=1}^{N} \pi_{i j} P_{j} x(t) \\
& \quad+x^{T}(t) Q x(t)-x^{T}(t-d) Q x(t-d) \\
& \quad+d^{2} x^{T}(t) A_{i}^{T} Z A_{i} x(t)-\int_{t-d}^{t} \dot{x}^{T}(\theta) Z \dot{x}(\theta) d \theta \\
& \mathscr{L} V_{2}\left(x_{t}\right) \\
&=e^{T}(t)\left[P_{i}\left(A_{i}-G_{i} C_{i}\right)\right]^{\star} e(t) \\
& \quad+2 \bar{\alpha} e^{T}(t) P_{i} G_{i} C_{i} x(t) \\
& \quad-2 \bar{\alpha} e^{T}(t) P_{i} G_{i} C_{i} x(t-d)+2 \alpha e^{T}(t) P_{i} G_{i} C_{i} \eta \\
&+2 \bar{\alpha} e^{T}(t) P_{i} G_{i} C_{i} \eta_{d}+e^{T}(t) \sum_{j=1}^{N} \pi_{i j} P_{j} e(t) \\
&+\alpha \bar{\alpha} d^{2}\left[G_{i} C_{i}\left(x(t-d)-x(t)+\eta-\eta_{d}\right)\right]^{T} \\
&+\int_{t-d}^{t} \dot{e}^{T}(\theta) Z \dot{e}(\theta) d \theta+e^{T}(t) Q e(t) \\
&-e^{T}(t-d) Q e(t-d) \\
&\left.\left.+d^{2}\left(f_{i 1}+f_{i 2}\right)^{T} Z(t-d)-x(t)+\eta-\eta_{d}\right)\right] \\
& \\
& \\
&
\end{aligned}
$$


where

$$
\begin{aligned}
f_{i 1}= & \left(A_{i}-G_{i} C_{i}\right) e(t)+\bar{\alpha} G_{i} C_{i} x(t) \\
& -\bar{\alpha} G_{i} C_{i} x(t-d), \\
f_{i 2}= & \alpha G_{i} C_{i} \eta+\bar{\alpha} G_{i} C_{i} \eta_{d} .
\end{aligned}
$$

Based on the Jensen inequality, one has

$$
\begin{aligned}
& -\int_{t-d}^{t} \dot{x}^{T}(\theta) Z \dot{x}(\theta) d \theta \\
& \leq-x^{T}(t) Z x(t)+2 x^{T}(t) Z x(t-d) \\
& \quad-x^{T}(t-d) Z x(t-d), \\
& -\int_{t-d}^{t} \dot{e}^{T}(\theta) Z \dot{e}(\theta) d \theta \\
& \leq-e^{T}(t) Z e(t)+2 e^{T}(t) Z e(t-d) \\
& \quad-e^{T}(t-d) Z e(t-d) .
\end{aligned}
$$

Moreover, it is concluded that

$$
\begin{aligned}
& 2 \alpha e^{T}(t) P_{i} G_{i} C_{i} \eta \leq \alpha e^{T}(t) P_{i} e(t) \\
& \quad+\alpha \eta^{T} C_{i}^{T} G_{i}^{T} P_{i} G_{i} C_{i} \eta \\
& 2 \bar{\alpha} e^{T}(t) P_{i} G_{i} C_{i} \eta_{d} \leq \bar{\alpha} e^{T}(t) P_{i} e(t) \\
& \quad+\bar{\alpha} \eta_{d}^{T} C_{i}^{T} G_{i}^{T} P_{i} G_{i} C_{i} \eta_{d}, \\
& 2 d^{2}\left(\alpha G_{i} C_{i} \eta+\bar{\alpha} G_{i} C_{i} \eta_{d}\right)^{T} Z\left(\alpha G_{i} C_{i} \eta+\bar{\alpha} G_{i} C_{i} \eta_{d}\right) \\
& \quad \leq 2 \alpha d^{2} \eta^{T} C_{i}^{T} G_{i}^{T} Z G_{i} C_{i} \eta+2 \bar{\alpha} d^{2} \eta_{d}^{T} C_{i}^{T} G_{i}^{T} Z G_{i} C_{i} \eta_{d} \\
& 2 \alpha \bar{\alpha} d^{2}\left(G_{i} C_{i} \eta-G_{i} C_{i} \eta_{d}\right)^{T} Z\left(G_{i} C_{i} \eta-G_{i} C_{i} \eta_{d}\right) \\
& \quad \leq 4 \alpha \bar{\alpha} d^{2} \eta^{T} C_{i}^{T} G_{i}^{T} Z G_{i} C_{i} \eta \\
& \quad+4 \alpha \bar{\alpha} d^{2} \eta_{d}^{T} C_{i}^{T} G_{i}^{T} Z G_{i} C_{i} \eta_{d} \\
& d^{2}\left(f_{i 1}+f_{i 2}\right)^{T} Z\left(f_{i 1}+f_{i 2}\right) \leq 2 d^{2} f_{i 1}^{T} Z f_{i 1} \\
& \quad+2 d^{2}\left(\alpha G_{i} C_{i} \eta+\bar{\alpha} G_{i} C_{i} \eta_{d}\right)^{T} \\
& \quad \cdot Z\left(\alpha G_{i} C_{i} \eta+\bar{\alpha} G_{i} C_{i} \eta_{d}\right) \\
& \alpha \bar{\alpha} d^{2}\left[G_{i} C_{i}\left(x(t-d)-x(t)+\eta-\eta_{d}\right)\right]^{T} \\
& \quad \cdot Z\left[G_{i} C_{i}\left(x(t-d)-x(t)+\eta-\eta_{d}\right)\right] \\
& \quad \leq 2 \alpha \bar{\alpha} d^{2}\left[G_{i} C_{i}(x(t-d)-x(t))\right]^{T} \\
& +Z\left[G_{i} C_{i}(x(t-d)-x(t))\right] \\
& +2 \alpha \bar{\alpha} d^{2}\left(G_{i} C_{i} \eta-G_{i} C_{i} \eta_{d}\right)^{T} Z\left(G_{i} C_{i} \eta-G_{i} C_{i} \eta_{d}\right)
\end{aligned}
$$

Based on the above conditions and applying Proposition 2, it is obtained that (37) is implied by

$$
\begin{gathered}
\mathscr{L} V\left(x_{t}\right) \leq \xi^{T}(t)\left(\left[\begin{array}{cccc}
\Theta_{i 1} & \Theta_{i 2} & Z & 0 \\
* & \Theta_{i 5} & \Theta_{i 6} & Z \\
* & * & \Theta_{i 8} & 0 \\
* & * & * & \Theta_{11}
\end{array}\right]\right. \\
+2 \alpha \bar{\alpha} d^{2}\left[\begin{array}{c}
-C_{i}^{T} G_{i}^{T} \\
0 \\
C_{i}^{T} G_{i}^{T} \\
0
\end{array}\right] Z\left[\begin{array}{c}
-C_{i}^{T} G_{i}^{T} \\
0 \\
C_{i}^{T} G_{i}^{T} \\
0
\end{array}\right]^{T}
\end{gathered}
$$

Moreover, the other conditions are assumed to be

$$
\begin{aligned}
& C_{i}^{T} G_{i}^{T} P_{i} G_{i} C_{i} \leq \gamma_{i} I, \\
& C_{i}^{T} G_{i}^{T} Z G_{i} C_{i} \leq \lambda_{i} I .
\end{aligned}
$$




$$
\left.+2 d^{2}\left[\begin{array}{c}
\bar{\alpha} C_{i}^{T} G_{i}^{T} \\
\left(A_{i}-C_{i}^{T} G_{i}\right)^{T} \\
-\bar{\alpha} C_{i}^{T} G_{i}^{T} \\
0
\end{array}\right] Z\left[\begin{array}{c}
\bar{\alpha} C_{i}^{T} G_{i}^{T} \\
\left(A_{i}-C_{i}^{T} G_{i}\right)^{T} \\
-\bar{\alpha} C_{i}^{T} G_{i}^{T} \\
0
\end{array}\right]^{T}\right)
$$

$\cdot \xi(t)$

where

$$
\xi^{T}(t)=\left[\begin{array}{llll}
x^{T}(t) & e^{T}(t) & x^{T}(t-d) & e^{T}(t-d)
\end{array}\right] .
$$

By using the Schur complement lemma, it is further obtained that

$$
\left[\begin{array}{cccccc}
\Theta_{i 1} & \Theta_{i 2} & Z & 0 & \bar{\Theta}_{i 3} & \bar{\Theta}_{i 4} \\
* & \Theta_{i 5} & \Theta_{i 6} & Z & 0 & \bar{\Theta}_{i 7} \\
* & * & \Theta_{i 8} & 0 & \bar{\Theta}_{i 9} & \bar{\Theta}_{i 10} \\
* & * & * & \Theta_{11} & 0 & 0 \\
* & * & * & * & -Z^{-1} & 0 \\
* & * & * & * & * & -Z^{-1}
\end{array}\right]<0,
$$

where

$$
\begin{aligned}
\bar{\Theta}_{i 3} & =-\sqrt{2 \alpha \bar{\alpha}} d C_{i}^{T} G_{i}^{T}, \\
\bar{\Theta}_{i 4} & =\sqrt{2} \bar{\alpha} d C_{i}^{T} G_{i}^{T}, \\
\bar{\Theta}_{i 9} & =\sqrt{2 \alpha \bar{\alpha}} d C_{i}^{T} G_{i}^{T}, \\
\bar{\Theta}_{i 7} & =\sqrt{2} d\left(A_{i}^{T}-C_{i}^{T} G_{i}^{T}\right), \\
\bar{\Theta}_{i 10} & =-\sqrt{2} \bar{\alpha} d C_{i}^{T} G_{i}^{T} .
\end{aligned}
$$

By pre- and postmultiplying both its sides with $\operatorname{diag}\{I, I, I$, $\left.I, P_{i}, P_{i}\right\}$ and its transpose, respectively, one gets

$$
\left[\begin{array}{cccccc}
\Theta_{i 1} & \Theta_{i 2} & Z & 0 & \Theta_{i 3} & \Theta_{i 4} \\
* & \Theta_{i 5} & \Theta_{i 6} & Z & 0 & \Theta_{i 7} \\
* & * & \Theta_{i 8} & 0 & \Theta_{i 9} & \Theta_{i 10} \\
* & * & * & \Theta_{11} & 0 & 0 \\
* & * & * & * & -\bar{Z} & 0 \\
* & * & * & * & * & -\bar{Z}
\end{array}\right]<0,
$$

where $\bar{Z}=P_{i} Z^{-1} P_{i}$. It is further obtained that $\bar{Z}>0$ could be guaranteed by

$$
-P_{i} Z^{-1} P_{i} \leq-2 P_{i}+Z<0
$$

As for conditions (38) and (39), they are equivalent to

$$
\begin{aligned}
& -\gamma_{i} I+C_{i}^{T} G_{i}^{T} P_{i} G_{i} C_{i} \leq 0, \\
& -\lambda_{i} I+C_{i}^{T} G_{i}^{T} Z G_{i} C_{i} \leq 0 .
\end{aligned}
$$

By applying the Schur complement lemma and considering representation (23), it is known that condition (38) is equivalent to condition (20). As for condition (39), by pre- and postmultiplying both its sides with $\operatorname{diag}\left\{I, P_{i}\right\}$ and applying inequality (45), one could easily get condition (21) with representation (23) implying condition (39). This completes the proof.

Remark 4. Due to $\alpha$ included obviously, it plays important roles in analysis and synthesis of systems with output saturation. With two special cases that only system state with $\alpha=0$ or delay state with $\alpha=1$ is contained in output saturation, our results could be viewed as extension results on the saturation output problem from output without time delay to stochastic delay output. On the other hand, from the proof process, it is seen that some inequalities, like (31), (33), (35), and so on, have been used to get the LMI conditions and could lead to more conservatism. In order to reduce its conservatism, some additional variables could be introduced in these inequalities. However, such variables will lead to larger computation complexity. Moreover, the conservatism of inequality (45) could be further reduced by applying similar methods in [45]. Similarly, much larger computation complexity in terms of more variables and inequalities should be needed. Based on these facts, it is said that whether to choose these methods or not should be considered in concrete situations.

Remark 5. It is seen from this theorem that such a probability should be given exactly. It will be impossible or of high cost in some practical applications. Instead, only its estimation is available, even if it is totally unknown. In other words, there will be a uncertainty between the real and estimated values. As we know, such a uncertainty will degrade the system performance and even lead to unstable system. So, it is necessary and meaningful to consider this general case. Moreover, some necessarily additional variables and inequalities are introduced to obtain LMI conditions, where the variables are to be computed instead of being given beforehand. Because of the given results with LMI forms, the above general case could be handled by exploiting the methods in this paper and $[46,47]$ together.

Next, we consider another state observer system described as

$$
\begin{aligned}
& \dot{\hat{x}}(t)=A\left(r_{t}\right) \hat{x}(t)+G\left(r_{t}\right)(y(t)-\widehat{y}(t)), \\
& \widehat{y}(t)=C\left(r_{t}\right)[\alpha(t) \hat{x}(t)+(1-\alpha(t)) \widehat{x}(t-d)] .
\end{aligned}
$$

Then, the resulting error system is rewritten to be

$$
\begin{aligned}
\dot{e}(t)=( & \left.A\left(r_{t}\right)-\alpha G\left(r_{t}\right) C\left(r_{t}\right)\right) e(t)-(1-\alpha) G\left(r_{t}\right) \\
\cdot & C\left(r_{t}\right) e(t-d)+\alpha G\left(r_{t}\right) C\left(r_{t}\right) \eta+(1-\alpha) \\
\cdot & G\left(r_{t}\right) C\left(r_{t}\right) \eta_{d}+(\alpha(t)-\alpha) G\left(r_{t}\right) C\left(r_{t}\right) \\
\cdot & \left(x(t-d)-x(t)+\eta-\eta_{d}\right) .
\end{aligned}
$$

Similarly, we have the following theorem. 
Theorem 6. Given system (1) with $d>0$, there exists observer (47) such that system (1) and error system (48) are asymptotically stable, if for given positive scalars $\alpha \in[0,1]$, $\varepsilon_{i} \in(0,1)$, and $\varepsilon_{d i} \in(0,1)$, there exist $P_{i}>0, Q>0, Z>$ $0, \gamma_{i}>0, \lambda_{i}>0$, and $Y_{i}$ satisfying conditions (20) and (21) and

$$
\left[\begin{array}{cccccc}
\Theta_{i 1} & 0 & Z & 0 & 0 & 0 \\
* & \Psi_{i 1} & 0 & \Psi_{i 2} & \Theta_{i 3} & \Psi_{i 3} \\
* & * & \Theta_{i 8} & 0 & 0 & 0 \\
* & * & * & \Theta_{11} & \Theta_{i 9} & \Theta_{i 10} \\
* & * & * & * & \Theta_{i 12} & 0 \\
* & * & * & * & * & \Theta_{i 12}
\end{array}\right]<0
$$

where

$$
\begin{aligned}
& \Psi_{i 1}=\left(P_{i} A_{i}-\alpha Y_{i} C_{i}\right)^{\star}+P_{i}+\sum_{j=1}^{N} \pi_{i j} P_{j}+Q-Z, \\
& \Psi_{i 2}=-\bar{\alpha} Y_{i} C_{i}+Z, \\
& \Psi_{i 3}=\sqrt{2} d\left(A_{i}^{T} P_{i}-\alpha C_{i}^{T} Y_{i}^{T}\right) .
\end{aligned}
$$

Then, the gain of observer (47) could be computed by (23).

Proof. For systems (1) and (48), choose the same Lyapunov function (24). Similar to (35) and (36), it is obtained that

$$
\begin{aligned}
& d^{2}\left(f_{i 3}+f_{i 2}\right)^{T} Z\left(f_{i 3}+f_{i 2}\right) \leq 2 d^{2} f_{i 3}^{T} Z f_{i 3} \\
& \quad+2 d^{2}\left(\alpha G_{i} C_{i} \eta+\bar{\alpha} G_{i} C_{i} \eta_{d}\right)^{T} \\
& \quad \cdot Z\left(\alpha G_{i} C_{i} \eta+\bar{\alpha} G_{i} C_{i} \eta_{d}\right),
\end{aligned}
$$

where

$$
\begin{aligned}
f_{i 3} & =\left(A_{i}-\alpha G_{i} C_{i}\right) e(t)-\bar{\alpha} G_{i} C_{i} e(t-d), \\
\alpha \bar{\alpha} & d^{2}\left[G_{i} C_{i}\left(e(t-d)-e(t)+\eta-\eta_{d}\right)\right]^{T} \\
& \cdot Z\left[G_{i} C_{i}\left(e(t-d)-e(t)+\eta-\eta_{d}\right)\right] \\
\leq & 2 \alpha \bar{\alpha} d^{2}\left[G_{i} C_{i}(e(t-d)-e(t))\right]^{T} \\
& \cdot Z\left[G_{i} C_{i}(e(t-d)-e(t))\right] \\
+ & 2 \alpha \bar{\alpha} d^{2}\left(G_{i} C_{i} \eta-G_{i} C_{i} \eta_{d}\right)^{T} Z\left(G_{i} C_{i} \eta-G_{i} C_{i} \eta_{d}\right) .
\end{aligned}
$$

Then, it is obtained that

$$
\begin{aligned}
\mathscr{L} V & \left(x_{t}\right) \leq x^{T}(t)\left(P_{i} A_{i}\right)^{\star} x(t)+x^{T}(t) \sum_{j=1}^{N} \pi_{i j} P_{j} x(t) \\
+ & e^{T}(t)\left[P_{i}\left(A_{i}-G_{i} C_{i}\right)\right]^{\star} e(t)-2 \bar{\alpha} e^{T}(t) \\
& \cdot P_{i} G_{i} C_{i} e(t-d)+e^{T}(t) P_{i} e(t) \\
+ & \alpha \eta^{T} C_{i}^{T} G_{i}^{T} P_{i} G_{i} C_{i} \eta+\bar{\alpha} \eta_{d}^{T} C_{i}^{T} G_{i}^{T} P_{i} G_{i} C_{i} \eta_{d}+e^{T}(t) \\
& \cdot \sum_{j=1}^{N} \pi_{i j} P_{j} e(t)+x^{T}(t) Q x(t)-x^{T}(t-d)
\end{aligned}
$$

$$
\begin{aligned}
& \cdot Q x(t-d)+e^{T}(t) Q e(t)-e^{T}(t-d) Q e(t-d) \\
& +d^{2} x^{T}(t) A_{i}^{T} Z A_{i} x(t)-x^{T}(t) Z x(t)+2 x^{T}(t) \\
& \cdot Z x(t-d)-x^{T}(t-d) Z x(t-d) \\
& +2 \alpha d^{2} \eta^{T} C_{i}^{T} G_{i}^{T} Z G_{i} C_{i} \eta+2 \bar{\alpha} d^{2} \eta_{d}^{T} C_{i}^{T} G_{i}^{T} Z G_{i} C_{i} \eta_{d} \\
& +2 d^{2}\left[\left(A_{i}-\alpha G_{i} C_{i}\right) e(t)-\bar{\alpha} G_{i} C_{i} e(t-d)\right]^{T} \\
& \times Z\left[\left(A_{i}-\alpha G_{i} C_{i}\right) e(t)-\bar{\alpha} G_{i} C_{i} e(t-d)\right] \\
& +2 \alpha \bar{\alpha} d^{2}\left[G_{i} C_{i}(e(t-d)-e(t))\right]^{T} \\
& \cdot Z\left[G_{i} C_{i}(e(t-d)-e(t))\right] \\
& +4 \alpha \bar{\alpha} d^{2} \eta^{T} C_{i}^{T} G_{i}^{T} Z G_{i} C_{i} \eta \\
& +4 \alpha \bar{\alpha} d^{2} \eta_{d}^{T} C_{i}^{T} G_{i}^{T} Z G_{i} C_{i} \eta_{d}-e^{T}(t) Z e(t)+2 e^{T}(t) \\
& \cdot Z e(t-d)-e^{T}(t-d) Z e(t-d) .
\end{aligned}
$$

Based on conditions (20), (21), (51), and (53), it is obtained that (54) is implied by

$$
\mathscr{L} V\left(x_{t}\right) \leq \xi^{T}(t)\left(\left[\begin{array}{cccc}
\Theta_{i 1} & 0 & Z & 0 \\
* & \Psi_{i 1} & 0 & \Psi_{i 2} \\
* & * & \Theta_{i 8} & 0 \\
* & * & * & \Theta_{11}
\end{array}\right]\right.
$$

$$
\begin{gathered}
+2 \alpha \bar{\alpha} d^{2}\left[\begin{array}{c}
0 \\
-C_{i}^{T} G_{i}^{T} \\
0 \\
C_{i}^{T} G_{i}^{T}
\end{array}\right] Z\left[\begin{array}{c}
0 \\
-C_{i}^{T} G_{i}^{T} \\
0 \\
C_{i}^{T} G_{i}^{T}
\end{array}\right]^{T} \\
\left.+2 d^{2}\left[\begin{array}{c}
\left(A_{i}-\alpha C_{i}^{T} G_{i}\right)^{T} \\
0 \\
-\bar{\alpha} C_{i}^{T} G_{i}^{T}
\end{array}\right] Z\left[\begin{array}{c}
0 \\
\left(A_{i}-\alpha C_{i}^{T} G_{i}\right)^{T} \\
0 \\
-\bar{\alpha} C_{i}^{T} G_{i}^{T}
\end{array}\right]\right)
\end{gathered}
$$

$\cdot \xi(t)$.

By using similar methods given in Theorem 3, it is known that condition (55) could be guaranteed by inequality (49). This completes the proof.

Finally, we consider the following state observer system described by

$$
\begin{aligned}
& \dot{\hat{x}}(t)=A\left(r_{t}\right) \hat{x}(t)+G\left(r_{t}\right)(y(t)-\widehat{y}(t)), \\
& \widehat{y}(t)=C\left(r_{t}\right) \text { sat }(\alpha(t) \hat{x}(t)+(1-\alpha(t)) \hat{x}(t-d))
\end{aligned}
$$


which is equivalent to

$$
\begin{aligned}
\dot{\hat{x}}(t) & =A\left(r_{t}\right) \hat{x}(t)+G\left(r_{t}\right)(y(t)-\widehat{y}(t)), \\
\widehat{y}(t) & =C\left(r_{t}\right)\{\alpha \widehat{x}(t)+\bar{\alpha} \widehat{x}(t-d) \\
+ & (\alpha(t)-\alpha)(\widehat{x}(t)-\widehat{x}(t-d)) \\
- & {\left.\left[\alpha \widehat{\eta}+\bar{\alpha} \widehat{\eta}_{d}+(\alpha(t)-\alpha)\left(\widehat{\eta}-\widehat{\eta}_{d}\right)\right]\right\} . }
\end{aligned}
$$

Then, the resulting error system is rewritten to be

$$
\begin{aligned}
\dot{e}(t)=( & \left.A\left(r_{t}\right)-\alpha G\left(r_{t}\right) C\left(r_{t}\right)\right) e(t)-\bar{\alpha} G\left(r_{t}\right) C\left(r_{t}\right) \\
& \cdot e(t-d)+\alpha G\left(r_{t}\right) C\left(r_{t}\right)(\eta-\widehat{\eta})+\bar{\alpha} G\left(r_{t}\right) \\
& \cdot C\left(r_{t}\right)\left(\eta_{d}-\widehat{\eta}_{d}\right)+(\alpha(t)-\alpha) G\left(r_{t}\right) C\left(r_{t}\right) \\
\cdot & \left(x(t-d)-x(t)+\eta-\widehat{\eta}-\eta_{d}+\widehat{\eta}_{d}\right) .
\end{aligned}
$$

Theorem 7. Given system (1) with $d>0$, there exists observer (56) such that system (1) and error system (58) are asymptotically stable, iffor given positive scalars $\alpha \in[0,1], \varepsilon_{i} \in$ $(0,1)$, and $\varepsilon_{d i} \in(0,1)$, there exist $P_{i}>0, Q>0, Z>0, \gamma_{i}>$ $0, \lambda_{i}>0$, and $Y_{i}$ satisfying conditions (20) and (21) and

$$
\left[\begin{array}{cccccc}
\Omega_{i 1} & \Omega_{i 2} & Z & 0 & 0 & 0 \\
* & \Omega_{i 3} & 0 & \Psi_{i 2} & \Theta_{i 3} & \Psi_{i 3} \\
* & * & \Omega_{i 4} & \Omega_{i 5} & 0 & 0 \\
* & * & * & \Omega_{i 6} & \Theta_{i 9} & \Theta_{i 10} \\
* & * & * & * & \Theta_{i 12} & 0 \\
* & * & * & * & * & \Theta_{i 12}
\end{array}\right]<0
$$

where

$$
\begin{aligned}
\Omega_{i 1}= & \left(P_{i} A_{i}\right)^{\star}+\sum_{j=1}^{N} \pi_{i j} P_{j}+2 \alpha \varepsilon_{i} \gamma_{i} I+Q+d^{2} A_{i}^{T} Z A_{i} \\
& -Z+8 \alpha d^{2} \varepsilon_{i} \lambda_{i} I+16 \alpha \bar{\alpha} d^{2} \varepsilon_{i} \lambda_{i} I, \\
\Omega_{i 2}= & -\alpha \varepsilon_{i} \gamma_{i} I-4 \alpha d^{2} \varepsilon_{i} \lambda_{i} I-8 \alpha \bar{\alpha} d^{2} \varepsilon_{i} \lambda_{i} I, \\
\Omega_{i 3}= & \left(P_{i} A_{i}-\alpha Y_{i} C_{i}\right)^{\star}+2 P_{i}+\sum_{j=1}^{N} \pi_{i j} P_{j}+Q-Z \\
& +\alpha \varepsilon_{i} \gamma_{i} I+4 \alpha d^{2} \varepsilon_{i} \lambda_{i} I+8 \alpha \bar{\alpha} d^{2} \varepsilon_{i} \lambda_{i} I, \\
\Omega_{i 4}= & 2 \bar{\alpha} \varepsilon_{d i} \gamma_{i} I+8 \bar{\alpha} d^{2} \varepsilon_{d i} \lambda_{i} I+16 \alpha \bar{\alpha} d^{2} \varepsilon_{d i} \lambda_{i} I-Q \\
& -Z, \\
\Omega_{i 5}= & -\bar{\alpha} \varepsilon_{d i} \gamma_{i} I-4 \bar{\alpha} d^{2} \varepsilon_{d i} \lambda_{i} I-8 \alpha \bar{\alpha} d^{2} \varepsilon_{d i} \lambda_{i} I, \\
\Omega_{i 6}= & \bar{\alpha} \varepsilon_{d i} \gamma_{i} I+4 \bar{\alpha} d^{2} \varepsilon_{d i} \lambda_{i} I+8 \alpha \bar{\alpha} d^{2} \varepsilon_{d i} \lambda_{i} I-Q-Z .
\end{aligned}
$$

Then, (23) could be used to compute the gain of observer (56).
Proof. Firstly, the same Lyapunov function is also selected for systems (1) and (56). Similar to computation process of Theorems 3 and 6, the corresponding terms are handled as

$$
\begin{aligned}
& -2 \alpha e^{T}(t) P_{i} G_{i} C_{i} \widehat{\eta} \\
& \leq \alpha e^{T}(t) P_{i} e(t)+\alpha \widehat{\eta}^{T} C_{i}^{T} G_{i}^{T} P_{i} G_{i} C_{i} \widehat{\eta}, \\
& -2 \bar{\alpha} e^{T}(t) P_{i} G_{i} C_{i} \widehat{\eta}_{d} \\
& \leq \bar{\alpha} e^{T}(t) P_{i} e(t)+\bar{\alpha} \widehat{\eta}_{d}^{T} C_{i}^{T} G_{i}^{T} P_{i} G_{i} C_{i} \widehat{\eta}_{d}, \\
& d^{2}\left(f_{i 3}+f_{i 4}\right)^{T} Z\left(f_{i 3}+f_{i 4}\right) \\
& \leq 2 d^{2} f_{i 3}^{T} Z f_{i 3}+2 d^{2} f_{i 4}^{T} Z f_{i 4},
\end{aligned}
$$

where

$$
\begin{aligned}
f_{i 4} & =\alpha G_{i} C_{i}(\eta-\widehat{\eta})+\bar{\alpha} G_{i} C_{i}\left(\eta_{d}-\widehat{\eta_{d}}\right) \\
2 d^{2} f_{i 4}^{T} Z f_{i 4} \leq 2 \alpha d^{2}(\eta-\widehat{\eta})^{T} C_{i}^{T} G_{i}^{T} Z G_{i} C_{i}(\eta-\widehat{\eta}) & +2 \bar{\alpha} d^{2}\left(\eta_{d}-\widehat{\eta_{d}}\right)^{T} C_{i}^{T} G_{i}^{T} Z G_{i} C_{i}\left(\eta_{d}-\widehat{\eta_{d}}\right) \\
& \leq 4 \alpha d^{2} \eta^{T} C_{i}^{T} G_{i}^{T} Z C_{i}^{T} G_{i}^{T} \eta+4 \alpha d^{2} \widehat{\eta}^{T} C_{i}^{T} G_{i}^{T} Z G_{i} C_{i} \widehat{\eta} \\
& +4 \bar{\alpha} d^{2} \eta_{d}^{T} C_{i}^{T} G_{i}^{T} Z G_{i} C_{i} \eta_{d}+4 \bar{\alpha} d^{2} \widehat{\eta}_{d}^{T} C_{i}^{T} G_{i}^{T} Z G_{i} C_{i} \widehat{\eta}_{d} \\
d^{2} & \left(f_{i 3}+f_{i 4}\right)^{T} Z\left(f_{i 3}+f_{i 4}\right) \leq 2 d^{2} f_{i 3}^{T} Z f_{i 3} \\
& +2 d^{2} f_{i 4}^{T} Z f_{i 4}, \\
\alpha \bar{\alpha} & d^{2}\left\{G_{i} C_{i}\left[e(t-d)-e(t)+(\eta-\widehat{\eta})-\left(\eta_{d}-\widehat{\eta_{d}}\right)\right]\right\}^{T} \\
& \times Z\left\{G_{i} C_{i}\left[e(t-d)-e(t)+(\eta-\widehat{\eta})-\left(\eta_{d}-\widehat{\eta_{d}}\right)\right]\right\} \\
& \leq 2 \alpha \bar{\alpha} d^{2}\left[G_{i} C_{i}(e(t-d)-e(t))\right]^{T} \\
& +Z\left[G_{i} C_{i}(e(t-d)-e(t))\right]+2 \alpha \bar{\alpha} d^{2}\left[G_{i} C_{i}(\eta-\widehat{\eta})\right. \\
& \left.-G_{i} C_{i}\left(\eta_{d}-\widehat{\eta_{d}}\right)\right]^{T} Z \times\left[G_{i} C_{i}(\eta-\widehat{\eta})-G_{i} C_{i}\left(\eta_{d}\right.\right. \\
& \left.\left.-\widehat{\eta_{d}}\right)\right] .
\end{aligned}
$$

Moreover, it is obtained that

$$
\begin{aligned}
2 \alpha \bar{\alpha} & d^{2}\left[G_{i} C_{i}(\eta-\widehat{\eta})-G_{i} C_{i}\left(\eta_{d}-\widehat{\eta_{d}}\right)\right]^{T} \\
\cdot & Z\left[G_{i} C_{i}(\eta-\widehat{\eta})-G_{i} C_{i}\left(\eta_{d}-\widehat{\eta_{d}}\right)\right] \\
& \leq 8 \alpha \bar{\alpha} d^{2} \eta^{T} C_{i}^{T} G_{i}^{T} Z C_{i}^{T} G_{i}^{T} \eta \\
& +8 \alpha \bar{\alpha} d^{2} \widehat{\eta}^{T} C_{i}^{T} G_{i}^{T} Z G_{i} C_{i} \widehat{\eta} \\
& +8 \alpha \bar{\alpha} d^{2} \eta_{d}^{T} C_{i}^{T} G_{i}^{T} Z G_{i} C_{i} \eta_{d} \\
& +8 \alpha \bar{\alpha} d^{2} \widehat{\eta}_{d}^{T} C_{i}^{T} G_{i}^{T} Z G_{i} C_{i} \widehat{\eta}_{d} .
\end{aligned}
$$


Based on these conditions, it is obtained that

$$
\begin{aligned}
& \mathscr{L} V\left(x_{t}\right) \leq x^{T}(t)\left(P_{i} A_{i}\right)^{\star} x(t)+x^{T}(t) \sum_{j=1}^{N} \pi_{i j} P_{j} x(t) \\
& +e^{T}(t)\left[P_{i}\left(A_{i}-G_{i} C_{i}\right)\right]^{\star} e(t)-2 \bar{\alpha} e^{T}(t) \\
& \text { - } P_{i} G_{i} C_{i} e(t-d)+2 e^{T}(t) P_{i} e(t) \\
& +\alpha \eta^{T} C_{i}^{T} G_{i}^{T} P_{i} G_{i} C_{i} \eta+\bar{\alpha} \eta_{d}^{T} C_{i}^{T} G_{i}^{T} P_{i} G_{i} C_{i} \eta_{d} \\
& +\alpha \widehat{\eta}^{T} C_{i}^{T} G_{i}^{T} P_{i} G_{i} C_{i} \widehat{\eta}+\bar{\alpha} \widehat{\eta}_{d}^{T} C_{i}^{T} G_{i}^{T} P_{i} G_{i} C_{i} \widehat{\eta}_{d}+e^{T}(t) \\
& \cdot \sum_{j=1}^{N} \pi_{i j} P_{j} e(t)+x^{T}(t) Q x(t)-x^{T}(t-d) \\
& \text { - } Q x(t-d)+e^{T}(t) Q e(t)-e^{T}(t-d) Q e(t-d) \\
& +d^{2} x^{T}(t) A_{i}^{T} Z A_{i} x(t)-x^{T}(t) Z x(t)+2 x^{T}(t) \\
& \cdot Z x(t-d)-x^{T}(t-d) Z x(t-d) \\
& +4 \alpha d^{2} \eta^{T} C_{i}^{T} G_{i}^{T} Z G_{i} C_{i} \eta+4 \bar{\alpha} d^{2} \eta_{d}^{T} C_{i}^{T} G_{i}^{T} Z G_{i} C_{i} \eta_{d} \\
& +4 \alpha d^{2} \widehat{\eta}^{T} C_{i}^{T} G_{i}^{T} Z G_{i} C_{i} \widehat{\eta}+4 \bar{\alpha} d^{2} \widehat{\eta}_{d}^{T} C_{i}^{T} G_{i}^{T} Z G_{i} C_{i} \widehat{\eta}_{d} \\
& +2 d^{2}\left[\left(A_{i}-\alpha G_{i} C_{i}\right) e(t)-\bar{\alpha} G_{i} C_{i} e(t-d)\right]^{T} \\
& \times Z\left[\left(A_{i}-\alpha G_{i} C_{i}\right) e(t)-\bar{\alpha} G_{i} C_{i} e(t-d)\right] \\
& +2 \alpha \bar{\alpha} d^{2}\left[G_{i} C_{i}(e(t-d)-e(t))\right]^{T} \\
& \cdot Z\left[G_{i} C_{i}(e(t-d)-e(t))\right] \\
& +8 \alpha \bar{\alpha} d^{2} \eta^{T} C_{i}^{T} G_{i}^{T} Z G_{i} C_{i} \eta \\
& +8 \alpha \bar{\alpha} d^{2} \eta_{d}^{T} C_{i}^{T} G_{i}^{T} Z G_{i} C_{i} \eta_{d} \\
& +8 \alpha \bar{\alpha} d^{2} \widehat{\eta}^{T} C_{i}^{T} G_{i}^{T} Z G_{i} C_{i} \widehat{\eta} \\
& +8 \alpha \bar{\alpha} d^{2} \widehat{\eta}_{d}^{T} C_{i}^{T} G_{i}^{T} Z G_{i} C_{i} \widehat{\eta}_{d}-e^{T}(t) Z e(t)+2 e^{T}(t) \\
& \text {. } Z e(t-d)-e^{T}(t-d) Z e(t-d) \text {. }
\end{aligned}
$$

Based on conditions (20) and (21) and above inequalities, it is obtained that (64) is implied by

$$
\begin{gathered}
\mathscr{L} V\left(x_{t}\right) \leq \xi^{T}(t)\left(\left[\begin{array}{cccc}
\Omega_{i 1} & \Omega_{i 2} & Z & 0 \\
* & \Omega_{i 3} & 0 & \Psi_{i 3} \\
* & * & \Omega_{i 4} & \Omega_{i 5} \\
* & * & * & \Omega_{i 6}
\end{array}\right]\right. \\
+2 \alpha \bar{\alpha} d^{2}\left[\begin{array}{c}
0 \\
-C_{i}^{T} G_{i}^{T} \\
0 \\
C_{i}^{T} G_{i}^{T}
\end{array}\right] Z\left[\begin{array}{c}
0 \\
-C_{i}^{T} G_{i}^{T} \\
0 \\
C_{i}^{T} G_{i}^{T}
\end{array}\right]^{T}
\end{gathered}
$$

$$
\left.+2 d^{2}\left[\begin{array}{c}
0 \\
\left(A_{i}-\alpha C_{i}^{T} G_{i}\right)^{T} \\
0 \\
-\bar{\alpha} C_{i}^{T} G_{i}^{T}
\end{array}\right] Z\left[\begin{array}{c}
0 \\
\left(A_{i}-\alpha C_{i}^{T} G_{i}\right)^{T} \\
0 \\
-\bar{\alpha} C_{i}^{T} G_{i}^{T}
\end{array}\right]^{T}\right)
$$$$
\cdot \xi(t)
$$

By using similar methods given in Theorems 3 and 6, condition (65) is obtained easily from inequality (59) with representation (23). This completes the proof.

\section{Numerical Examples}

Example 1. Consider a delayed Markovian jump system of form (1), whose parameters are described to be

$$
\begin{aligned}
& A_{1}=\left[\begin{array}{cc}
-1.5 & 0.1 \\
1 & -0.8
\end{array}\right], \\
& C_{1}=\left[\begin{array}{ll}
0.1 & 0.6 \\
0.4 & 0.1
\end{array}\right], \\
& A_{2}=\left[\begin{array}{ll}
-0.9 & -0.2 \\
0.8 & -0.9
\end{array}\right], \\
& C_{2}=\left[\begin{array}{ll}
-0.2 & 0.5 \\
-0.3 & -0.2
\end{array}\right] .
\end{aligned}
$$

The transition rate matrix is given as

$$
\Pi=\left[\begin{array}{cc}
-0.5 & 0.5 \\
0.6 & -0.6
\end{array}\right] \text {. }
$$

Firstly, we design an observer with form (9). Letting $d=0.3$, $\varepsilon_{1}=0.3, \varepsilon_{2}=0.2, \varepsilon_{d 1}=0.4$, and $\varepsilon_{d 2}=0.1$, by Theorem 3 , the gains of observer (9) are computed as

$$
\begin{aligned}
& G_{1}=\left[\begin{array}{ll}
0.0902 & 0.2853 \\
0.2443 & 0.2980
\end{array}\right], \\
& G_{2}=\left[\begin{array}{rr}
-0.1308 & -0.0389 \\
-0.0230 & -0.4683
\end{array}\right] .
\end{aligned}
$$

Applying the above designed observer, under the initial condition $x_{0}=\left[\begin{array}{llll}1 & -1 & -1 & 1\end{array}\right]^{T}$, we have the state responses of systems (1) and (10) given in Figure 1, while the simulation of the corresponding operation mode is given in Figure 2.

When another observer (47) is designed, its gains could be computed by Theorem 6 and given as

$$
\begin{aligned}
& G_{1}=\left[\begin{array}{ll}
0.0855 & 0.3848 \\
0.3415 & 0.2380
\end{array}\right], \\
& G_{2}=\left[\begin{array}{ll}
-0.1079 & -0.0338 \\
-0.0148 & -0.5234
\end{array}\right],
\end{aligned}
$$




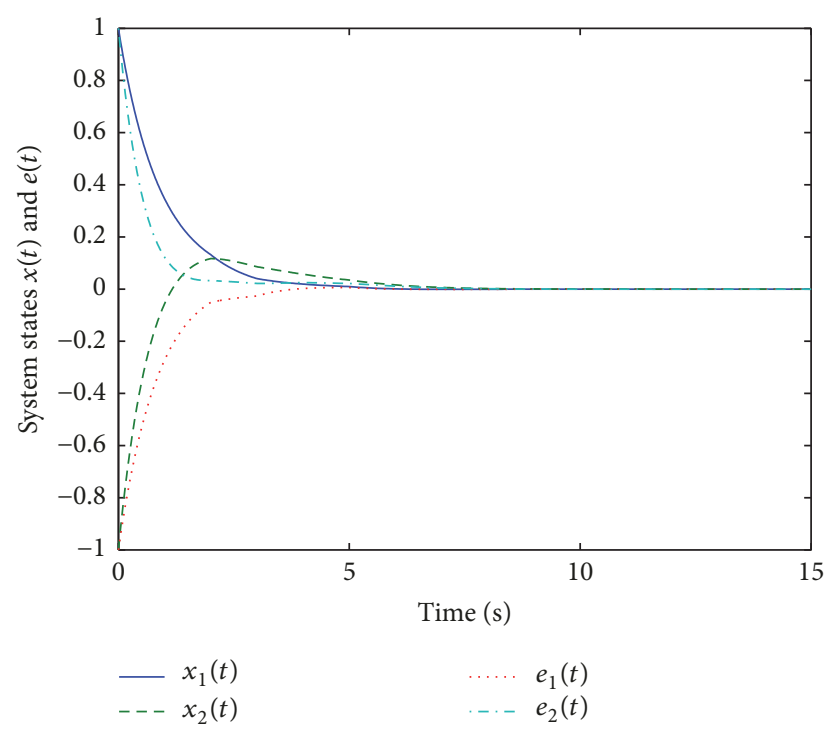

FIGURE 1: The state responses of systems (1) and (10).

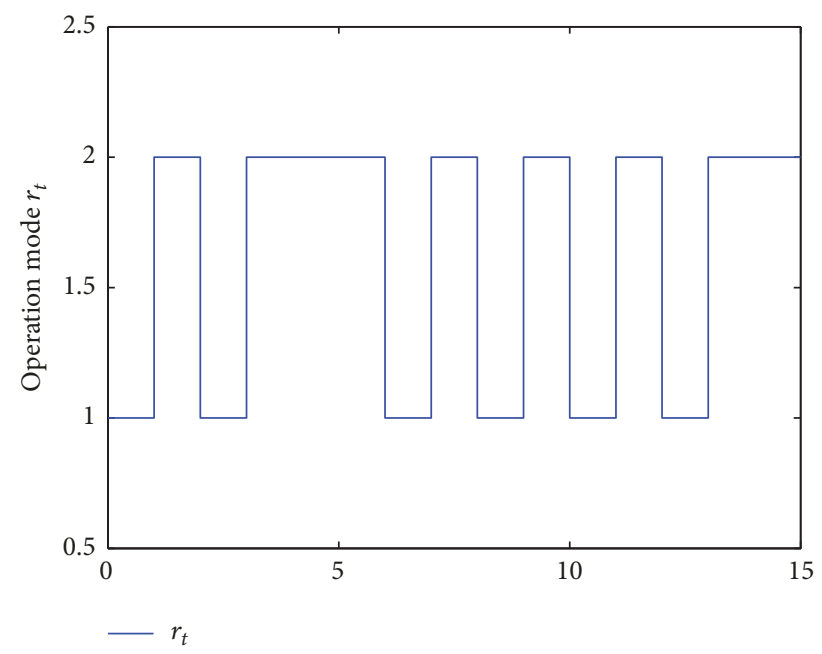

FIgURE 2: The simulation of operation mode.

where the corresponding parameters are the same as the above ones. At the same time, by Theorem 7, the gains of observer (57) are computed to be

$$
\begin{aligned}
& G_{1}=\left[\begin{array}{ll}
0.2832 & 0.9648 \\
0.2265 & 0.1486
\end{array}\right], \\
& G_{2}=\left[\begin{array}{cc}
0.1489 & 0.8130 \\
-0.3814 & -2.0525
\end{array}\right] .
\end{aligned}
$$

Under the same initial condition, the state curves of the corresponding systems are simulated in Figures 3 and 4, respectively. Based on these simulations, it is seen that the designed observers are all useful and also demonstrate the utility of the proposed methods.

In order to further demonstrate the effects of output saturation existing in different forms, more comparisons among

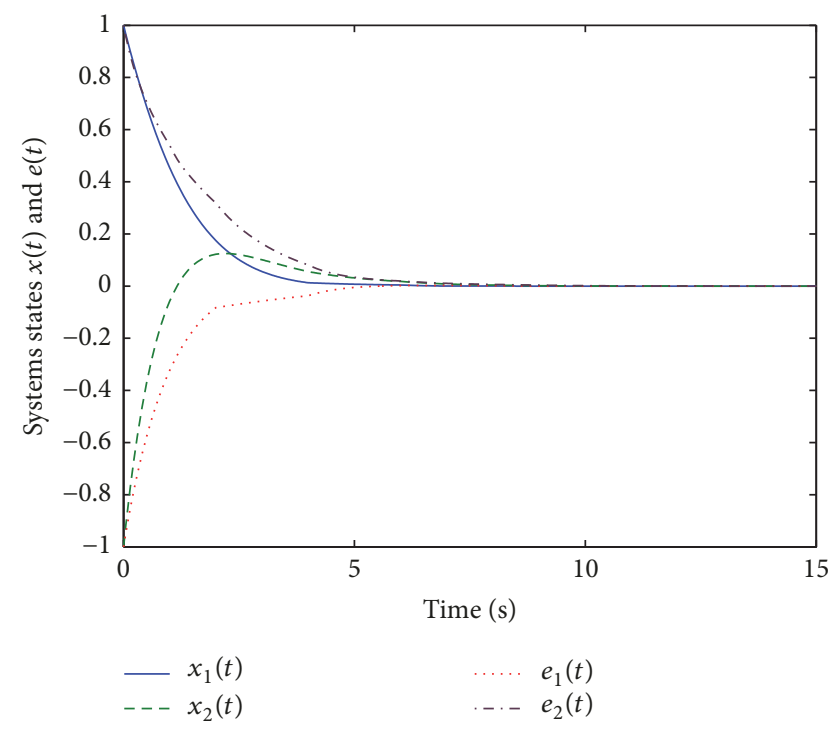

FIgURE 3: The state responses of systems (1) and (47).

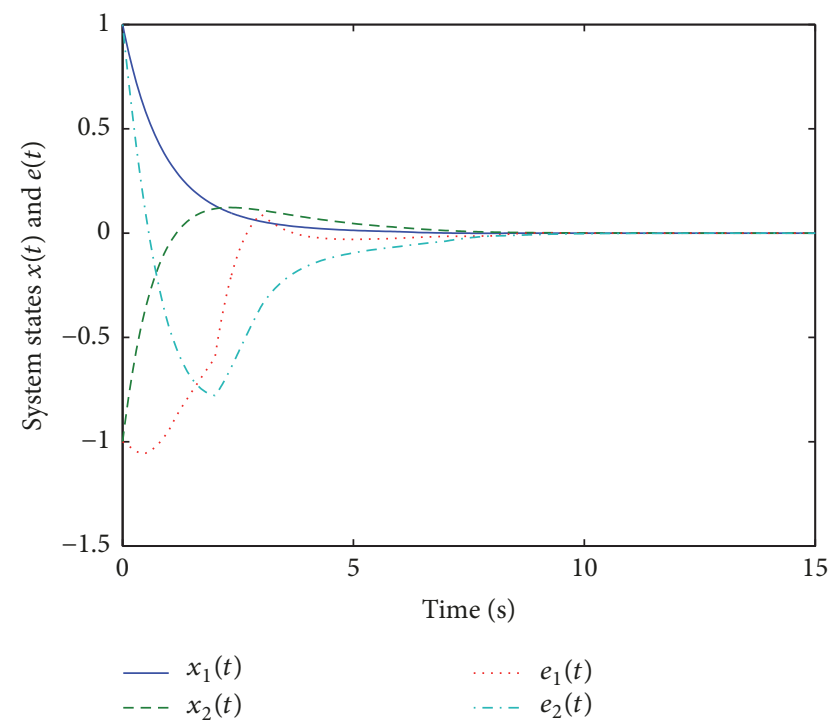

FIGURE 4: The state responses of systems (1) and (57).

them will be done in the following. Firstly, we consider the relationship between probability $\alpha$ and allowable maximum $d$. Based on Theorems 3-7, the maximum allowable $d$ along with $\alpha$ is given in Table 1 , whose simulation is carried out in Figure 5. From this simulation, it is seen that observer (9) is the least conservative, while observer (56) has the largest conservatism.

Next, we consider the effects of parameters $\varepsilon_{i}$ and $\varepsilon_{d i}$. In order to demonstrate their effects clearly, without loss of generality, matrix $A_{1}$ is assumed to be

$$
A_{1}=\left[\begin{array}{cc}
-1 & 0.1+\delta \\
1 & -0.8
\end{array}\right],
$$

where $\delta$ is a positive scalar to be determined, and the other matrices are constant. Based on Theorem 3, the allowable 
TABLE 1: The allowable maximum $d$ for different $\alpha$.

\begin{tabular}{lcccrr}
\hline$\alpha$ & 0 & 0.3 & 0.5 & 0.8 & 0.976 \\
\hline Theorem 3 & 0.927 & 0.943 & 0.955 & 0.999 \\
Theorem 6 & 0.918 & 0.928 & 0.936 & 0.958 & 0.999 \\
Theorem 7 & 0.207 & 0.273 & 0.313 & 0.390 & 0.470 \\
\hline
\end{tabular}

TABLE 2: The allowable $\delta_{\max }$ for different pair $\left(\varepsilon_{1}, \varepsilon_{2}\right)$.

\begin{tabular}{lcccc}
\hline & $\varepsilon_{2}=0.1$ & $\varepsilon_{2}=0.4$ & $\varepsilon_{2}=0.6$ & $\varepsilon_{2}=0.9$ \\
\hline$\varepsilon_{1}=0.1$ & 0.9450 & 0.9370 & 0.9340 & 0.9300 \\
$\varepsilon_{1}=0.4$ & 0.9000 & 0.8930 & 0.8900 & 0.8860 \\
$\varepsilon_{1}=0.6$ & 0.8760 & 0.8690 & 0.8650 & 0.8610 \\
$\varepsilon_{1}=0.9$ & 0.8440 & 0.8370 & 0.8340 & 0.8300 \\
\hline
\end{tabular}

TABLE 3: The allowable $\delta_{\max }$ for different pair $\left(\varepsilon_{1}, \varepsilon_{2}\right)$.

\begin{tabular}{lcccc}
\hline & $\varepsilon_{2}=0.1$ & $\varepsilon_{2}=0.4$ & $\varepsilon_{2}=0.6$ & $\varepsilon_{2}=0.9$ \\
\hline$\varepsilon_{1}=0.1$ & 0.9390 & 0.9330 & 0.9300 & 0.9260 \\
$\varepsilon_{1}=0.4$ & 0.8950 & 0.8900 & 0.8870 & 0.8830 \\
$\varepsilon_{1}=0.6$ & 0.8710 & 0.8660 & 0.8630 & 0.8590 \\
$\varepsilon_{1}=0.9$ & 0.8400 & 0.8350 & 0.8320 & 0.8280 \\
\hline
\end{tabular}

TABLE 4: The allowable $\delta_{\max }$ for different pair $\left(\varepsilon_{1}, \varepsilon_{2}\right)$.

\begin{tabular}{ccccc}
\hline & $\varepsilon_{2}=0.1$ & $\varepsilon_{2}=0.4$ & $\varepsilon_{2}=0.6$ & $\varepsilon_{2}=0.9$ \\
\hline$\varepsilon_{1}=0.01$ & 0.1960 & 0.1220 & 0.0870 & 0.0430 \\
$\varepsilon_{1}=0.05$ & 0.1690 & 0.1020 & 0.0690 & 0.0260 \\
$\varepsilon_{1}=0.10$ & 0.1410 & 0.0800 & 0.0480 & 0.0050 \\
$\varepsilon_{1}=0.40$ & 0.0390 & - & - \\
\hline
\end{tabular}

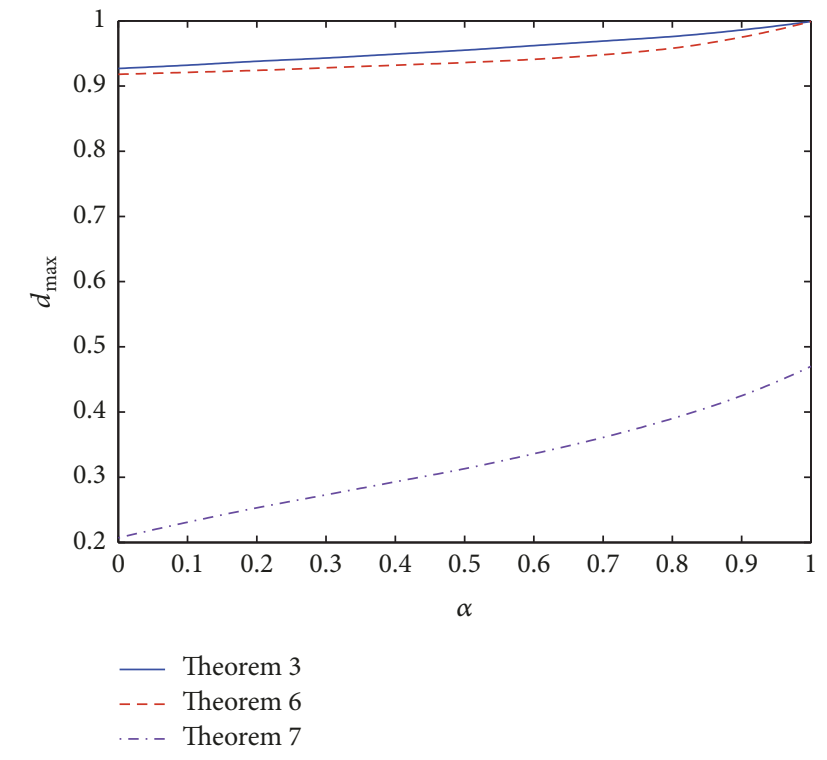

FIgURE 5: The curves of $d_{\max }$ along with $\alpha$.

maximum $\delta \triangleq \delta_{\max }$ along with different pair $\left(\varepsilon_{1}, \varepsilon_{2}\right)$ is listed in Table 2. Similarly, Tables 3 and 4 are obtained by applying Theorems 6 and 7, respectively. Based on these tables, the simulation of correlation between pair $\left(\varepsilon_{1}, \varepsilon_{2}\right)$ and $\delta_{\max }$ is shown Figures 6 and 7. From Figure 6, it is seen that Theorem 3 related to observer (10) is less conservative than Theorem 6 corresponding to observer (47). From their forms, it is seen in this example that the delay term in output has a negative effect. However, the differences between them are very small. To the contrary, there is an advantage that the nondelay state of observer (47) is not necessarily available online, which could be replaced by a delay one with some probability. In this sense, it is said that observer (47) is better in terms of having less application constraints. As for Figure 7, it is concluded that observer (57) is the most conservative. The main reason is that a more output saturation is included and has a large negative effect in reducing performance. Similarly, we could also make a correlation between pair $\left(\varepsilon_{d 1}, \varepsilon_{d 2}\right)$ and $\delta_{\max }$. Based on Theorems 3-7, the allowable $\delta_{\max }$ for different pair $\left(\varepsilon_{d 1}, \varepsilon_{d 2}\right)$ are presented in Tables 5-7. From these tables, a similar conclusion could be obtained where Theorem 3 designed for observer (10) is superior over Theorems 6 and 7. Among them, it is found that the most conservative one is observer (57) obtained from Theorem 7. In addition, the corresponding simulations of such correlations between pair $\left(\varepsilon_{d 1}, \varepsilon_{d 2}\right)$ and $\delta_{\max }$ could be obtained easily and will give the same conclusion, which are omitted here. 
TABLE 5: The allowable $\delta_{\max }$ for different pair $\left(\varepsilon_{d 1}, \varepsilon_{d 2}\right)$.

\begin{tabular}{lcccc}
\hline & $\varepsilon_{d 2}=0.1$ & $\varepsilon_{d 2}=0.4$ & $\varepsilon_{d 2}=0.6$ & $\varepsilon_{d 2}=0.9$ \\
\hline$\varepsilon_{d 1}=0.1$ & 0.9470 & 0.9420 & 0.9390 & 0.9350 \\
$\varepsilon_{d 1}=0.4$ & 0.9110 & 0.9080 & 0.9060 & 0.9030 \\
$\varepsilon_{d 1}=0.6$ & 0.8890 & 0.8860 & 0.8840 & 0.8820 \\
$\varepsilon_{d 1}=0.9$ & 0.8580 & 0.8550 & 0.8540 & 0.8510 \\
\hline
\end{tabular}

TABLE 6: The allowable $\delta_{\max }$ for different pair $\left(\varepsilon_{d 1}, \varepsilon_{d 2}\right)$.

\begin{tabular}{lcccc}
\hline & $\varepsilon_{d 2}=0.1$ & $\varepsilon_{d 2}=0.4$ & $\varepsilon_{d 2}=0.6$ & $\varepsilon_{d 2}=0.9$ \\
\hline$\varepsilon_{d 1}=0.1$ & 0.9440 & 0.9390 & 0.9370 & 0.9330 \\
$\varepsilon_{d 1}=0.4$ & 0.9060 & 0.9020 & 0.9000 & 0.8960 \\
$\varepsilon_{d 1}=0.6$ & 0.8830 & 0.8790 & 0.8770 & 0.8730 \\
$\varepsilon_{d 1}=0.9$ & 0.8510 & 0.8470 & 0.8450 & 0.8420 \\
\hline
\end{tabular}

TABLE 7: The allowable $\delta_{\max }$ for different pair $\left(\varepsilon_{d 1}, \varepsilon_{d 2}\right)$.

\begin{tabular}{ccccc}
\hline & $\varepsilon_{d 2}=0.1$ & $\varepsilon_{d 2}=0.4$ & $\varepsilon_{d 2}=0.6$ & $\varepsilon_{d 2}=0.9$ \\
\hline$\varepsilon_{d 1}=0.01$ & 0.1950 & 0.1490 & 0.1210 & 0.0850 \\
$\varepsilon_{d 1}=0.05$ & 0.1830 & 0.1380 & 0.1100 & 0.0730 \\
$\varepsilon_{d 1}=0.10$ & 0.1660 & 0.1220 & 0.0940 & 0.0570 \\
$\varepsilon_{d 1}=0.40$ & 0.0470 & 0.0110 & - & - \\
\hline
\end{tabular}

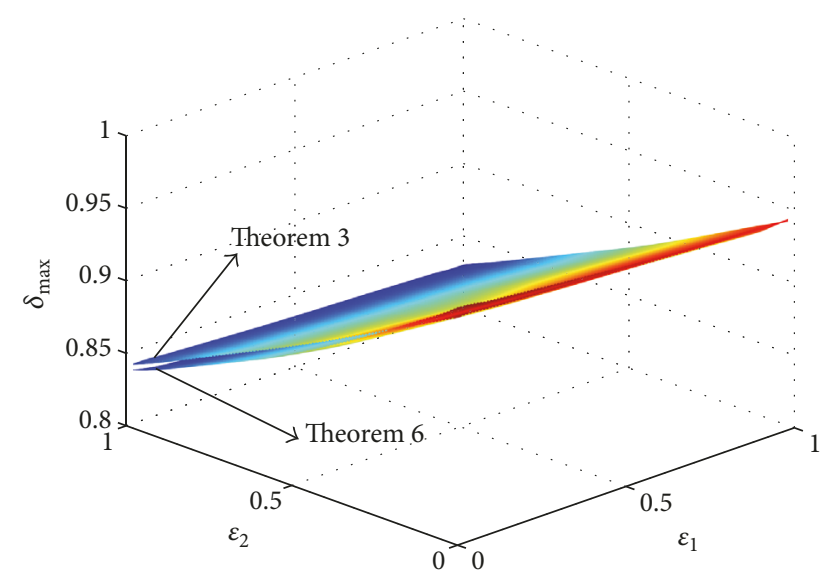

FIGURE 6: The simulation of correlation between pair $\left(\varepsilon_{1}, \varepsilon_{2}\right)$ and $\delta_{\max }$.

\section{Conclusion}

In this paper, we have studied observer design problem of continuous-time Markovian jump systems with saturated output. First of all, a kind of state observer with output state saturation is proposed to be partially dependent. More precisely, both nondelay and delay sates are contained but occur asynchronously, whose probability distributions are embodied by the Bernoulli variable and taken into account in the observer design. By applying an improved inequality to deal with saturation terms, the existence conditions for observers with three kinds of output state saturations have been proposed with LMIs. Finally, a numerical example is used to testify the effectiveness and advantages of the presented methods.

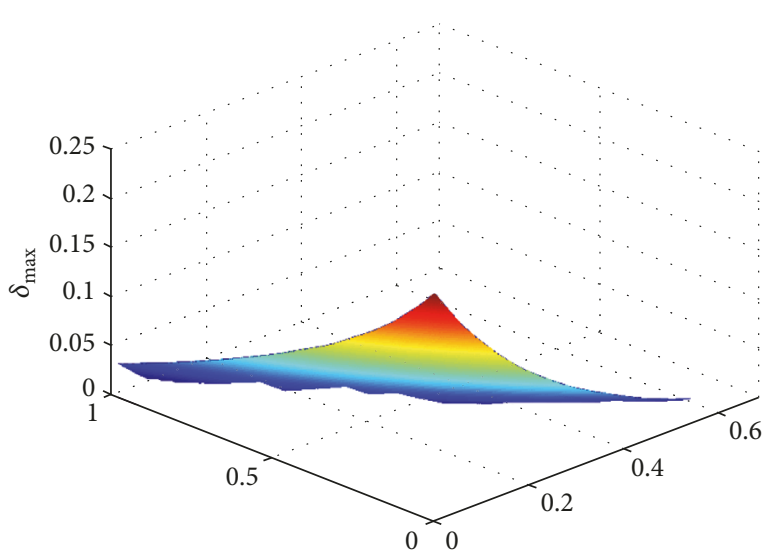

FIGURE 7: The simulation of correlation between pair $\left(\epsilon_{1}, \epsilon_{2}\right)$ and $\delta_{\max }$.

\section{Conflicts of Interest}

The authors declare that there are no conflicts of interest regarding the publication of this paper.

\section{Acknowledgments}

This work was supported by the National Natural Science Foundation of China under Grants 61374043 and 61473140, the Program for Liaoning Excellent Talents in University under Grant LJQ2013040, and the Natural Science Foundation of Liaoning Province under Grant 2014020106. 


\section{References}

[1] S.-L. Kong and Z.-S. Zhang, "Optimal control of stochastic system with Markovian jumping and multiplicative noises," Acta Automatica Sinica, vol. 38, no. 7, pp. 1113-1118, 2012.

[2] W. Li and Z. Wu, "Output tracking of stochastic high-order nonlinear systems with Markovian switching," Institute of Electrical and Electronics Engineers Transactions on Automatic Control, vol. 58, no. 6, pp. 1585-1590, 2013.

[3] S. Lakshmanan, F. A. Rihan, R. Rakkiyappan, and J. H. Park, "Stability analysis of the differential genetic regulatory networks model with time-varying delays and Markovian jumping parameters," Nonlinear Analysis: Hybrid Systems, vol. 14, pp. 115, 2014.

[4] J. Xiong, J. Lam, Z. Shu, and X. Mao, "Stability analysis of continuous-time switched systems with a random switching signal," Institute of Electrical and Electronics Engineers Transactions on Automatic Control, vol. 59, no. 1, pp. 180-186, 2014.

[5] J. Duan, M. Hu, Y. Yang, and L. Guo, "A delay-partitioning projection approach to stability analysis of stochastic Markovian jump neural networks with randomly occurred nonlinearities," Neurocomputing, vol. 128, pp. 459-465, 2014.

[6] C.-D. Zheng, Y. Zhang, and Z. Wang, "Stability analysis of stochastic reaction-diffusion neural networks with Markovian switching and time delays in the leakage terms," International Journal of Machine Learning and Cybernetics, vol. 5, no. 1, pp. 3-12, 2014.

[7] N. K. Kwon, B. Y. Park, and P. Park, "Less conservative stabilization conditions for Markovian jump systems with incomplete knowledge of transition probabilities and input saturation," Optimal Control Applications and Methods, vol. 37, no. 6, pp. 1207-1216, 2016.

[8] B. Y. Park, N. K. Kwon, and P. Park, "Stabilization of Markovian jump systems with incomplete knowledge of transition probabilities and input quantization," Journal of The Franklin Institute, vol. 352, no. 10, pp. 4354-4365, 2015

[9] Y. Kao, J. Xie, and C. Wang, "Stabilization of singular Markovian jump systems with generally uncertain transition rates," Institute of Electrical and Electronics Engineers Transactions on Automatic Control, vol. 59, no. 9, pp. 2604-2610, 2014.

[10] H. Fan, B. Liu, W. Wang, and C. Wen, "Adaptive fault-tolerant stabilization for nonlinear systems with Markovian jumping actuator failures and stochastic noises," Automatica, vol. 51, pp. 200-209, 2015.

[11] M. Shen, J. H. Park, and D. Ye, "A Separated Approach to Control of Markov Jump Nonlinear Systems with General Transition Probabilities," IEEE Transactions on Cybernetics, vol. 46, no. 9, pp. 2010-2018, 2016.

[12] P. Shi, Y. Yin, F. Liu, and J. Zhang, "Robust control on saturated Markov jump systems with missing information," Information Sciences, vol. 265, pp. 123-138, 2014.

[13] L. Qiu, Y. Shi, F. Q. Yao, G. Xu, and B. G. Xu, "Network-based robust control for linear system With two-channel random packet dropout and time delays," IEEE Transactions on Cybernetics, vol. 45, no. 8, pp. 1450-1462, 2015.

[14] H. Zhang, Y. Shi, and J. Wang, “Observer-based tracking controller design for networked predictive control systems with uncertain Markov delays," International Journal of Control, vol. 86, no. 10, pp. 1824-1836, 2013.

[15] E. Tian, D. Yue, and G. Wei, "Robust control for Markovian jump systems with partially known transition probabilities and nonlinearities," Journal of The Franklin Institute, vol. 350, no. 8, pp. 2069-2083, 2013.

[16] H. Li, P. Shi, D. Yao, and L. Wu, "Observer-based adaptive sliding mode control for nonlinear Markovian jump systems," Automatica, vol. 64, pp. 133-142, 2016.

[17] X. Zhong, H. He, H. Zhang, and Z. Wang, "Optimal control for unknown discrete-time nonlinear markov jump systems using adaptive dynamic programming," IEEE Transactions on Neural Networks and Learning Systems, vol. 25, no. 12, pp. 2141-2155, 2014.

[18] R. Chang, Y. Fang, L. Liu, and J. Li, "Decentralized prescribed performance adaptive tracking control for Markovian jump uncertain nonlinear systems with input saturation," International Journal of Adaptive Control and Signal Processing, vol. 31, no. 2, pp. 255-274, 2017.

[19] M. Liu, D. W. C. Ho, and P. Shi, "Adaptive fault-tolerant compensation control for Markovian jump systems with mismatched external disturbance," Automatica, vol. 58, pp. 5-14, 2015.

[20] M. Q. Shen, D. Ye, and Q. G. Wang, "Event-triggered H8 filtering of Markov jump systems with general transition probabilities," Information Sciences, vol. 418, pp. 635-651, 2017.

[21] H. Shen, L. Su, and J. H. Park, "Reliable mixed Ho passive control for T-S fuzzy delayed systems based on a semi-Markov jump model approach," Fuzzy Sets and Systems, vol. 314, pp. 7998, 2017.

[22] F. Li, L. Wu, P. Shi, and C.-C. Lim, "State estimation and sliding mode control for semi-Markovian jump systems with mismatched uncertainties," Automatica, vol. 51, pp. 385-393, 2015.

[23] P. Shi, Y. Zhang, and R. Agarwal, "Stochastic finite-time state estimation for discrete time-delay neural networks with Markovian jumps," Neurocomputing, vol. 151, part 1, pp. 168-174, 2014.

[24] H. Zhang and J. Wang, "State estimation of discrete-time Takagi-Sugeno fuzzy systems in a network environment," IEEE Transactions on Cybernetics, vol. 45, no. 8, pp. 1525-1536, 2014.

[25] J. Hu, D. Chen, and J. Du, "State estimation for a class of discrete nonlinear systems with randomly occurring uncertainties and distributed sensor delays," International Journal of General Systems, vol. 43, no. 3-4, pp. 387-401, 2014.

[26] Z. G. Wu, P. Shi, H. Su, and J. Chu, "Stochastic synchronization of Markovian jump neural networks with time-varying delay using sampled data," IEEE Transactions on Cybernetics, vol. 43, no. 6, pp. 1796-1806, 2013.

[27] X. Wang, J. A. Fang, Y. H, and A. D. Dai, "Finite-time global synchronization for a class of Markovian jump complex networks with partially unknown transition rates under feedback control," Nonlinear Dynamics, vol. 79, no. 1, pp. 47-61, 2015.

[28] C. Allefeld and S. Bialonski, "Detecting synchronization clusters in multivariate time series via coarse-graining of Markov chains," Physical Review E: Statistical, Nonlinear, and Soft Matter Physics, vol. 76, no. 6, Article ID 066207, 2007.

[29] Z.-X. Li, J. H. Park, and Z.-G. Wu, "Synchronization of complex networks with nonhomogeneous Markov jump topology," Nonlinear Dynamics, vol. 74, no. 1-2, pp. 65-75, 2013.

[30] H. Shen, J. H. Park, Z.-G. Wu, and Z. Zhang, "Finite-time Ho synchronization for complex networks with semi-Markov jump topology," Communications in Nonlinear Science and Numerical Simulation, vol. 24, no. 1-3, pp. 40-51, 2015.

[31] H. Michael, Z. Chen, X. Wang, and J. Lam, "Semi-global observer-based leader-following consensus with input saturation," IEEE Transactions on Industrial Electronics, vol. 61, no. 6, pp. 2842-2850, 2014. 
[32] Q. Zhang, C. Schaaf, and K. C. Seto, "The Vegetation adjusted NTL Urban Index: A new approach to reduce saturation and increase variation in nighttime luminosity," Remote Sensing of Environment, vol. 129, pp. 32-41, 2013.

[33] W. Sun, H. Gao, and O. Kaynak, "Vibration isolation for active suspensions with performance constraints and actuator saturation," IEEE/ASME Transactions on Mechatronics, vol. 20, no. 2, pp. 675-683, 2015.

[34] S. Gao, H. Dong, Y. Chen, B. Ning, G. Chen, and X. Yang, "Approximation-based robust adaptive automatic train control: an approach for actuator saturation," IEEE Transactions on Intelligent Transportation Systems, vol. 14, no. 4, pp. 1733-1742, 2013.

[35] H. Y. Li, J. H. Hui, and P. Shi, "Output-feedback based sliding mode control for fuzzy systems with actuator saturation," IEEE Transactions on Fuzzy Systems, vol. 24, no. 6, pp. 1282-1293, 2016.

[36] L. Sun, Y. Wang, and G. Feng, "Control design for a class of affine nonlinear descriptor systems with actuator saturation," Institute of Electrical and Electronics Engineers Transactions on Automatic Control, vol. 60, no. 8, pp. 2195-2200, 2015.

[37] J. Klamkin, Y.-C. Chang, A. Ramaswamy et al., "Output saturation and linearity of waveguide unitraveling-carrier photodiodes," IEEE Journal of Quantum Electronics, vol. 44, no. 4, pp. 354-359, 2008.

[38] J. Klamkin, A. Ramaswamy, L. A. Johansson et al., "High output saturation and high-linearity uni-traveling-carrier waveguide photodiodes," IEEE Photonics Technology Letters, vol. 19, no. 3, pp. 149-151, 2007.

[39] X. Ji, Y. Sun, and H. Su, "Analysis and design for singular discrete linear systems subject to actuator saturation," Asian Journal of Control, vol. 13, no. 2, pp. 350-355, 2011.

[40] H. Liu, E.-K. Boukas, F. Sun, and D. W. C. Ho, "Controller design for Markov jumping systems subject to actuator saturation," Automatica, vol. 42, no. 3, pp. 459-465, 2006.

[41] S. Ma and C. Zhang, "Ho control for discrete-time singular Markov jump systems subject to actuator saturation," Journal of The Franklin Institute, vol. 349, no. 3, pp. 1011-1029, 2012.

[42] C. A. C. Gonzaga and O. L. V. Costa, "Stochastic stabilization and induced $\ell 2$-gain for discrete-time Markov jump Lur'e systems with control saturation," Automatica, vol. 50, no. 9, pp. 2397-2404, 2014.

[43] L. Zhang, E.-K. Boukas, and A. Haidar, "Delay-range-dependent control synthesis for time-delay systems with actuator saturation," Automatica, vol. 44, no. 10, pp. 2691-2695, 2008.

[44] B. Y. Zhang, S. Y. Xu, Y. S. Zhang, and Y. M. Chu, "Delaydependent stabilization for delayed Markovian jump systems subject to input saturation," in Proceedings of the 29th Chinese Control Conference (CCC'10), pp. 5981-5986, Beijing, China, 2010.

[45] Y. S. Moon, P. Park, W. H. Kwon, and Y. S. Lee, "Delaydependent robust stabilization of uncertain state-delayed systems," International Journal of Control, vol. 74, no. 14, pp. 14471455, 2001.

[46] G. Wang, S. Xu, and Y. Zou, "Stabilisation of hybrid stochastic systems by disordered controllers," IET Control Theory \& Applications, vol. 8, no. 13, pp. 1154-1162, 2014.

[47] G. Wang, P. Zhang, and Q. Zhang, "A generalized robust Ho filtering for singular Markovian jump systems and its applications," International Journal of Robust and Nonlinear Control, vol. 24, no. 18, pp. 3491-3507, 2014. 


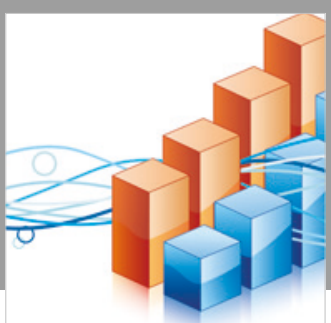

Advances in

Operations Research

\section{-n-m}
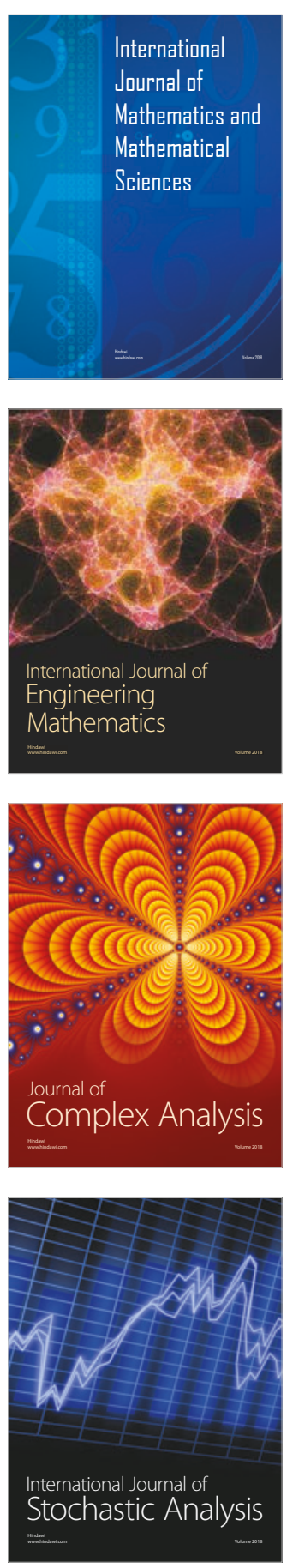
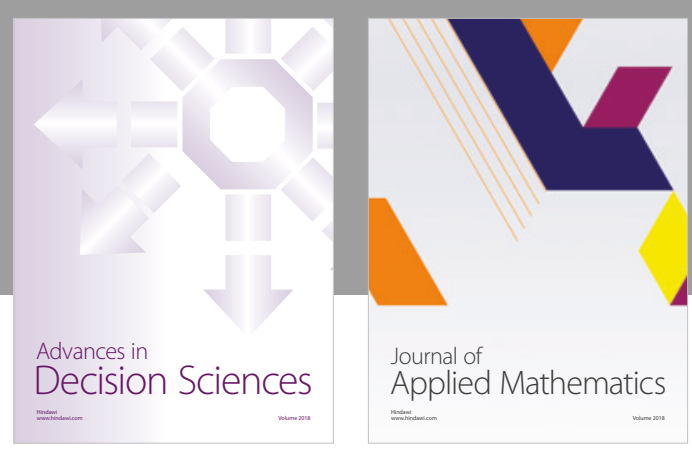

Journal of

Applied Mathematics
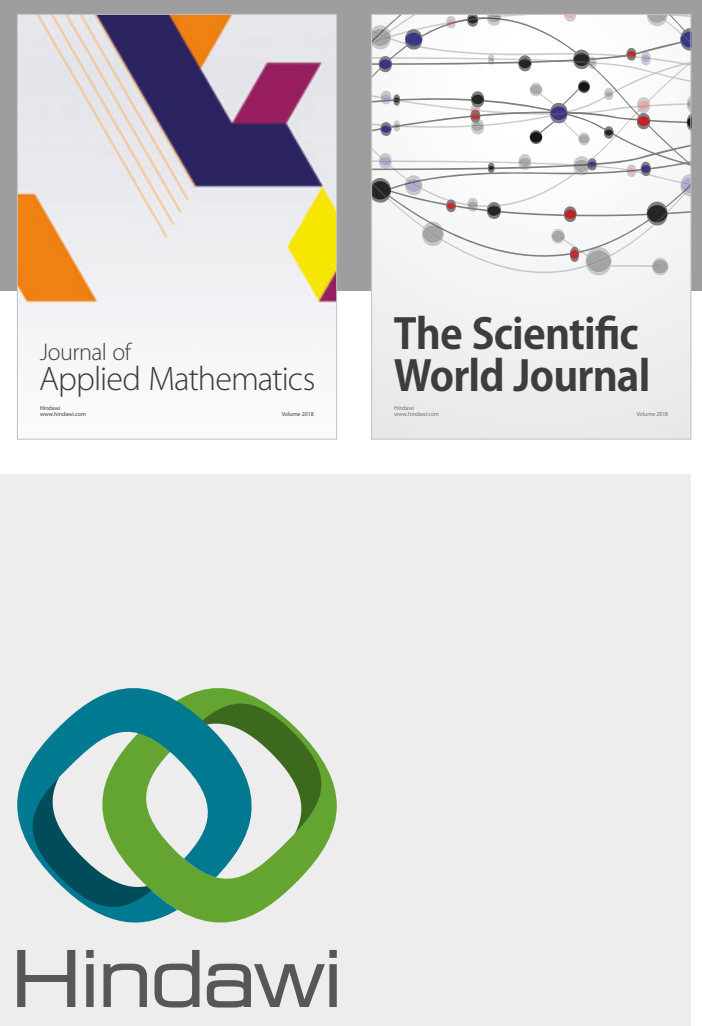

Submit your manuscripts at

www.hindawi.com

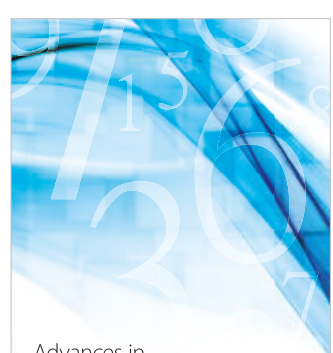

Advances in
Numerical Analysis
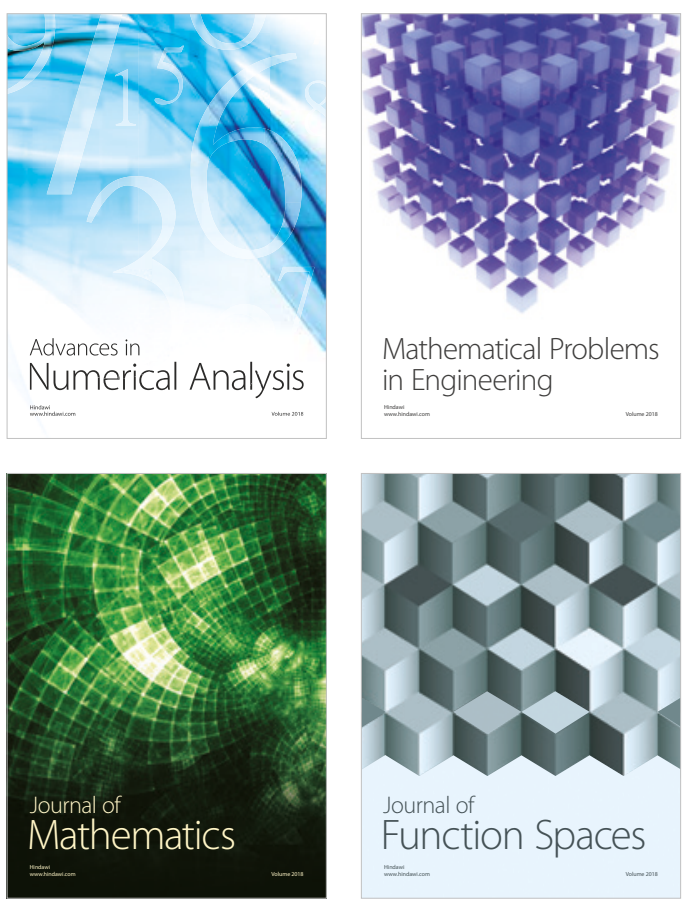

Mathematical Problems in Engineering

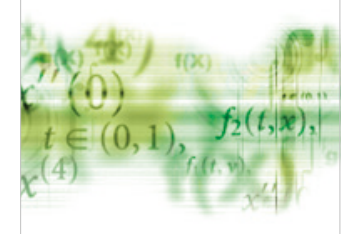

International Journal of

Differential Equations

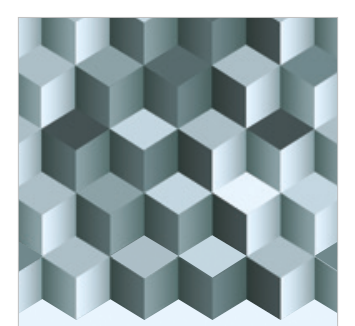

Journal of

Function Spaces

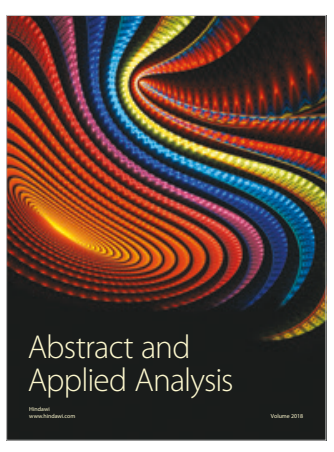

The Scientific

World Journal

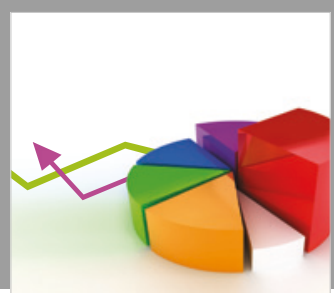

Journal of

Probability and Statistics
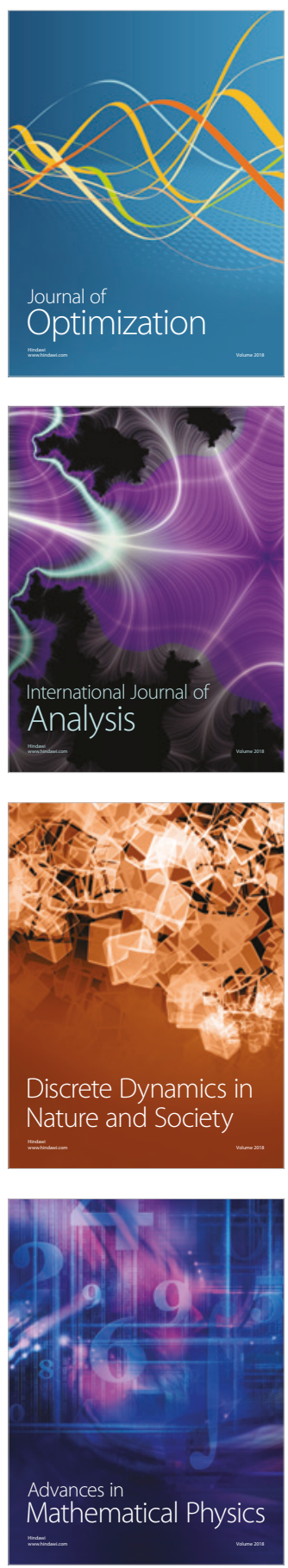